\title{
Inverse Portfolio Problem With Coherent Risk Measures
}

\author{
Bogdan Grechuk \\ Department of Mathematics, University of Leicester, LE1 7RH, UK \\ bg83@leicester.ac.uk 44(0116)252-5238 \\ Michael Zabarankin \\ Department of Mathematical Sciences, Stevens Institute of Technology, Hoboken, NJ, USA \\ mzabaran@stevens.edu 1(201)216-5434
}

\begin{abstract}
In general, a portfolio problem minimizes risk (or negative utility) of a portfolio of financial assets with respect to portfolio weights subject to a budget constraint. The inverse portfolio problem then arises when an investor assumes that his/her risk preferences have a numerical representation in the form of a certain class of functionals, e.g. in the form of expected utility, coherent risk measure or mean-deviation functional, and aims to identify such a functional, whose minimization results in a portfolio, e.g. a market index, that he/she is most satisfied with. In this work, the portfolio risk is determined by a coherent risk measure, and the rate of return of investor's preferred portfolio is assumed to be known. The inverse portfolio problem then recovers investor's coherent risk measure either through finding a convex set of feasible probability measures (risk envelope) or in the form of either mixed CVaR or negative Yaari's dual utility. It is solved in single-period and multi-period formulations and is demonstrated in a case study with the FTSE 100 index.
\end{abstract}

Key words: decision making under risk, coherent risk measure, portfolio optimization, inverse portfolio problem.

\section{Introduction}

Identifying agent's risk preferences has been and continues to be one of the main thrusts of decision making under uncertainty. Von Neumann and Morgenstern [34] were the first who proposed four axioms on a preference relation: completeness, transitivity, continuity, and independence and showed that these axioms admit a numerical representation in the form of expected utility. Due to its mathematical elegance and relative simplicity, the expected utility theory (EUT) still remains a normative model of the theory of choice despite continuing debate and criticism since its inception. On the one hand, empirical evidence (so-called paradoxes) shows that some of EUT's axioms are consistently violated (see, e.g., Allais paradox [1]), whereas, on the other hand, even if the four axioms are agreed to follow, an agent faces an exhaustive questionnaire procedure for identifying his/her utility function. A considerable number of alternative theories of choice have emerged only to encounter their own paradoxes and to reveal that they hardly have advantage over the EUT; see dual utility theory [31,35], weighted expected utility theory (WEUT) [7], rank-dependent (anticipated) utility theory (AUT) [25], prospect theory [9, 15], cumulative prospect theory (CPT) [16], regret theory [21], disappointment theory [5], mean-deviation theory [11], to mention just a few.

While all utility theories begin with axioms on a preference relation and then derive numerical representations for those axioms, the recently emerged theory of coherent risk measures [2] introduces the notion of acceptance set $\mathcal{A}$ for risky alternatives (random variables) and measures risk of a random variable (r.v.) $X$ as the minimum amount of cash $C$ that makes $X+C$ acceptable. In other words, a risk measure $\rho$ of $X$ can be determined as $\rho(X)=\min \{C \in \mathbb{R} \mid X+C \in \mathcal{A}\}$. If $\mathcal{A}$ is a closed positively homogeneous convex cone containing all nonnegative r.v.'s and no strictly negative r.v.'s, then $\rho$ is a positively homogeneous convex functional and is called coherent risk measure [2]. In this case, it admits a dual representation in the form $\rho(X)=\sup _{Q \in \mathcal{Q}} E[Q(-X)]$, where $\mathcal{Q}$ is a closed convex set whose elements are nonnegative r.v.'s with expected value of 1 (constant 1 is also included). The set $\mathcal{Q}$ is called risk envelope and each its element can be 
interpreted as a "distortion" of the underlined probability measure. Namely this interpretation ties risk preferences to a certain convex set of probability measures. In other words, the agent can specify "scenarios" of probability measures and can evaluate risk of an r.v. $X$ as the negative expected value of $X$ under the worst-case probability measure over the convex hull of the specified probability measures; see, e.g., [20]. In fact, there is a one-to-one relationship between coherent risk measures and risk envelopes.

Given the elegant risk-envelope characterization and the attractive properties (convexity, positive homogeneity, monotonicity, and constant translation) of coherent risk measures, the agent may reasonably assume that his/her risk preferences have a numerical representation in the form of a coherent risk measure $\rho$, which he/she would use in a variety of decision making problems: optimal portfolio selection [23], optimal risk sharing [14], optimal hedging [32], etc. Also, $-\rho(X)$ plays the role of a utility functional of an r.v. $X$, and the question of identifying $\rho(X)$ is equivalent to the one of identifying the utility functional $U(X)=-\rho(X)$. One of the approaches to finding $\rho$ is to solve the inverse portfolio problem: if the agent minimizes the coherent risk measure $\rho$ over some set of r.v.'s (portfolio returns), and $X^{*}$ is known to be optimal, i.e. the rate of return of an optimal portfolio, then $\rho$ can be recovered from $X^{*}$ through determining the risk envelope $\mathcal{Q}$ associated with $\rho$. This approach was demonstrated in identifying a deviation measure in a Markowitz-type portfolio selection problem; see [12] and [36, Section 8.2.4]. However, in general, the results in [12] cannot be used directly to solve the inverse portfolio problem with an unrestricted class of coherent risk measures, since deviation measures correspond one-to-one to averse measures of risk [27,28], ${ }^{1}$ which, in contrast to coherent risk measures, are not required to preserve monotonicity of preference relations; see [27, 28] and [36, Section 2.3]. In fact, these two classes of risk measures have a nonempty intersection called coherent averse measures of risk that correspond one-to-one to lower range dominated deviation measures (subclass of general deviation measures). For example, the standard deviation is not lower range dominated, and as a result, it has no counterpart in coherent risk measures through the one-to-one correspondence established in [27, 28]. Also, the work [12] assumes $X^{*}$ to be SSD-efficient to guarantee the existence of a law invariant solution, which is no longer required here.

This paper solves the inverse portfolio problem with coherent risk measures in both single-period and multiperiod formulations. In the single-period formulation, a set $\mathcal{X}$ of feasible portfolio rates of return at some time moment $t_{0}$ in the past is given, and the agent's optimal portfolio rate at $t_{0}$ is known to be $X^{*} \in \mathcal{X}$. The inverse problem is then finding a coherent risk measure $\rho$ from a given class $\mathcal{R}$, determined by some additional requirements/constraints on agent's risk preferences, such that $X^{*}$ is agent's optimal choice. It is shown that if a law-invariant solution to the inverse problem exists, it can always be represented in the form of mixed $\mathrm{CVaR}$, and a technique for explicitly computing that mixed CVaR is suggested. ${ }^{2}$ Moreover, if an exact solution does not exist, the technique returns the "best" approximate mixed CVaR. In the multi-period formulation, a sequence of agent's optimal portfolio rates of return is given (each of which is from a given feasible set), and $\rho \in \mathcal{R}$ is sought to make all those portfolio rates optimal (or at least close to optimal).

The rest of the paper is organized into four sections. Section 2 introduces coherent risk measures. Section 3 formulates and solves the inverse portfolio problem with coherent risk measures, whereas Section 4 constructs approximate solutions in the form of mixed CVaR. Section 5 concludes the work and proposes future research directions.

\section{Coherent Risk Measures}

Let $\mathcal{X}$ be a set of available random variables (r.v.'s), e.g. representing rates of return of risky assets. Formally, an r.v. is a measurable function from some probability space $\Omega=(\Omega, \mathcal{M}, \mathbb{P})$ to $\mathbb{R}$, where $\Omega$ is the set of future states $\omega, \mathcal{M}$ is a field of sets in $\Omega$, and $\mathbb{P}$ is a probability measure on $(\Omega, \mathcal{M})$. The relations between

\footnotetext{
${ }^{1}$ In $[27,28]$, they are originally called strictly expectation bounded risk measures.

${ }^{2}$ In [12], the technique for identifying deviation measures requires $X^{*}$ to have unique risk identifiers with respect to all CVaR's, which considerably limits the number of appropriate $X^{*}$ (especially of real-life instruments). Here, the corresponding technique works for arbitrary $X^{*}$.
} 
r.v.'s will be understood to hold in the almost sure sense, e.g., we write $X=Y$ if $\mathbb{P}[X=Y]=1$ and $X \geqslant Y$ if $\mathbb{P}[X \geqslant Y]=1$. By $F_{X}(x)$ and $q_{X}(\alpha)=\inf \left\{x \mid F_{X}(x)>\alpha\right\}$ will be meant the cumulative distribution function (CDF) and quantile function of an r.v. $X$, respectively. Let $\mathcal{L}^{\infty}(\Omega)=\mathcal{L}^{\infty}(\Omega, \mathcal{M}, \mathbb{P})$ be the space of all bounded r.v.'s with the norm $\|X\|_{\infty}=\sup |X|$, and let $\mathcal{L}^{1}(\Omega)=\mathcal{L}^{1}(\Omega, \mathcal{M}, \mathbb{P})$ be the space of all r.v.'s with finite expectation with the norm $\|X\|_{1}=E|X|$. The probability space $\Omega$ is called atomless, if there exists an r.v. with a continuous $\mathrm{CDF}^{3}$

We assume that every r.v. $X$ from $\mathcal{X}$ is bounded, and that an agent evaluates risk of $X$ by a risk measure $\rho: \mathcal{L}^{\infty}(\Omega) \rightarrow \mathbb{R}$ satisfying four axioms:

(i) Monotonicity: $\rho(X) \geqslant \rho(Y)$ whenever $X \leqslant Y$;

(ii) Sub-additivity: $\rho(X+Y) \leqslant \rho(X)+\rho(Y)$ for all $X, Y \in \mathcal{L}^{\infty}(\Omega)$;

(iii) Positive homogeneity: $\rho(\alpha X)=\alpha \rho(X)$ for every $X \in \mathcal{L}^{\infty}(\Omega)$ and $\alpha \geqslant 0$;

(iv) Translation property: $\rho(X+C)=\rho(X)-C$ for every $X \in \mathcal{L}^{\infty}(\Omega)$ and constant $C$.

A functional $\rho: \mathcal{L}^{\infty}(\Omega) \rightarrow \mathbb{R}$ satisfying (i)-(iv) is called coherent risk measure [2]. The set of all r.v.'s with non-positive risk, i.e.

$$
\mathcal{A}_{\rho}=\left\{X \in \mathcal{L}^{\infty}(\Omega) \mid \rho(X) \leqslant 0\right\}
$$

is called the acceptance set associated with $\rho$. The four axioms of $\rho$ imply that $\mathcal{A}_{\rho}$ is a positively homogeneous convex cone containing all nonnegative r.v.'s and no strictly negative r.v.'s; see [2]. In fact, $\mathcal{A}_{\rho}$ gives $\rho$ back as

$$
\rho(X)=\min _{C \in \mathbb{R}: X+C \in \mathcal{A}_{\rho}} C,
$$

which shows that $\rho(X)$ is the minimum amount of cash infusion needed for $X$ to be acceptable.

Every coherent risk measure satisfying the Fatou property ${ }^{4}$ can be represented by

$$
\rho(X)=\sup _{Q \in \mathcal{Q}} E[Q(-X)]
$$

for some $\mathcal{Q} \subset \mathcal{Q}^{M A X} \subset \mathcal{L}^{1}(\Omega)$, where

$$
\mathcal{Q}^{M A X}=\left\{Q \in \mathcal{L}^{1}(\Omega) \mid Q \geqslant 0, E[Q]=1\right\},
$$

see [2]. The set $\mathcal{Q}$ in (3) is called the risk envelope of $\rho$ and is recovered from $\rho$ as

$$
\mathcal{Q}=\left\{Q \in \mathcal{L}^{1}(\Omega) \mid E[Q(-X)] \leqslant \rho(X) \text { for all } X \in \mathcal{L}^{\infty}(\Omega)\right\} .
$$

Since every $Q \in \mathcal{Q}^{M A X}$ is a Radon-Nikodym derivative of some probability measure $\mathbb{P}^{Q}$ with respect to $\mathbb{P}$, $E[Q(-X)]=E_{\mathbb{P} Q}[-X]$ can be viewed as the expected loss associated with $X$ under $\mathbb{P}^{Q}$. The set $\mathcal{Q}$ in (3) can then be interpreted as the set of Radon-Nikodym derivatives of probability measures, or scenarios, which the agent considered "likely," and the risk measure in (3) is then interpreted as the average loss under the "worst" scenario. If the supremum in (3) is attained for some $Q$, it is called a risk identifier for $X$ [30]. The set of all risk identifiers for $X$ is denoted by $\mathcal{Q}_{\rho}(X)$.

The representations (1) and (3) imply that the acceptance set $\mathcal{A}_{\rho}$ can be determined by the risk envelope $\mathcal{Q}$ by

$$
\mathcal{A}_{\rho}=\left\{X \in \mathcal{L}^{\infty}(\Omega) \mid E[Q X] \geqslant 0 \text { for all } Q \in \mathcal{Q}\right\},
$$

\footnotetext{
${ }^{3}$ The existing literature offers several definitions of an atomless space, all of which are equivalent, see [8, Proposition A.27].

${ }^{4}$ A functional $\rho$ satisfies the Fatou property, if $\rho(X) \leqslant \lim \inf _{n \rightarrow \infty} \rho\left(X_{n}\right)$ for any bounded sequence $X_{n}$ with $X_{n} \rightarrow X$ a.s. Since $X_{n} \rightarrow X$ a.s. does not imply $X_{n} \rightarrow X$ in the $\mathcal{L}^{\infty}$ norm, this property is stronger than lower semicontinuity.
} 
whereas it follows from (2) and (4) that the risk envelope $\mathcal{Q}$ is "induced" by $\mathcal{A}_{\rho}$ as

$$
\mathcal{Q}=\left\{Q \in \mathcal{L}^{1}(\Omega) \mid E[Q]=1, E[Q X] \geqslant 0 \text { for all } X \in \mathcal{A}_{\rho}\right\}
$$

A coherent risk measure $\rho$ is called law-invariant if $\rho(X)=\rho(Y)$ whenever $X$ and $Y$ have the same distribution function. This is equivalent to the assumption that the risk envelope $\mathcal{Q}$ associated with $\rho$ is lawinvariant: $Q_{1} \in \mathcal{Q}$ implies that $Q_{2} \in \mathcal{Q}$ whenever $Q_{1}$ and $Q_{2}$ have the same distribution, see [6, Corollary 4.3].

The best known example of a law-invariant coherent risk measure is arguably conditional value-at-risk (CVaR) [26], defined for any $\alpha \in(0,1]$ by

$$
\operatorname{CVaR}_{\alpha}(X) \equiv-\frac{1}{\alpha} \int_{0}^{\alpha} q_{X}(\beta) d \beta
$$

By continuity, we can define

$$
\mathrm{CVaR}_{0}(X)=\lim _{\alpha \rightarrow 0} \mathrm{CVaR}_{\alpha}(X)=-\inf X .
$$

A straightforward generalization of CVaR is mixed CVaR [27,28]

$$
\rho(X)=\int_{0}^{1} \operatorname{CVaR}_{\alpha}(X) d \lambda(\alpha)
$$

where $\lambda$ is a weight function ${ }^{5}$ on $[0,1]$, i.e. $\lambda(\alpha) \geqslant 0$ and $\int_{0}^{1} d \lambda(\alpha)=1$. If $\lim _{\epsilon \rightarrow 0} \int_{0}^{\epsilon} d \lambda(\alpha)=0,{ }^{6}$ mixed CVaR (5) can be represented in the spectral form

$$
\rho(X)=-\int_{0}^{1} \phi(\alpha) q_{X}(\alpha) d \alpha
$$

where $\phi(\alpha)=\int_{[\alpha, 1)} s^{-1} d \lambda(s)$ is a left-continuous and nonincreasing function with $\phi\left(1^{-}\right)=0$ and $\int_{0}^{1} \phi(\alpha) d \alpha=$ 1; see Theorem 4.70 in [8]. Guriev [13, Theorem 1] showed that the integral in (6) (without negative sign) is exactly a Yaari's dual utility function [35].

Under a minor technical assumption, ${ }^{7}$ every law-invariant coherent risk measure can be represented as a worst-case mixed CVaR

$$
\rho(X)=\sup _{\lambda \in \Lambda} \int_{0}^{1} \mathrm{CVaR}_{\alpha}(X) d \lambda(\alpha)
$$

where $\Lambda$ is a collection of probability measures $\lambda$ on $[0,1]$, see Corollary 4.63 and Remark 4.64 in [8]. Equivalently, $\rho(X)$ in (7) can be represented as

$$
\rho(X)=\sup _{Q \in \mathcal{Q}} \int_{0}^{1} q_{Q}(\alpha) q_{-X}(\alpha) d \alpha,
$$

where $\mathcal{Q}$ is the same as in (3), see Theorem 4.59 in [8].

In this work, investors are assumed to be non-satiable and risk averse, and their choices are assumed to be consistent with risk orderings [22].

\footnotetext{
${ }^{5}$ In [27,28], $\lambda$ is a weight function on $(0,1)$. Following [8, p. 220], we extend the definition of mixed CVaR to include $C V a R_{0}(X)=$ $-\inf X$ and $\mathrm{CVaR}_{1}(X)=-E[X]$. In particular, $\lambda$ can be a linear combination of delta-functions at $\alpha_{1}<\cdots<\alpha_{n}$, in which case, mixed CVaR (5) simplifies to $\sum_{i=1}^{n} w_{i} \mathrm{CVaR}_{\alpha_{i}}(X)$, where $w_{1}, \ldots, w_{n}$ are positive weights summing up to 1.

${ }^{6}$ In other words, if $\lambda$ is not a $\delta$-function at 0 .

${ }^{7}$ Namely, this holds if $\Omega$ is atomless and $\mathcal{L}^{2}(\Omega)$ is separable. The representation (7) was originally proved in [18] under somewhat more restricting conditions.
} 
An r.v. $X$ dominates an r.v. $Y$ with respect to second-order stochastic dominance (SSD), and we write $X \succcurlyeq_{S S D} Y$, if $E[f(X)] \geqslant E[f(Y)]$ for every concave increasing function $f: \mathbb{R} \rightarrow \mathbb{R}$, or, equivalently, if

$$
\operatorname{CVaR}_{\alpha}(X) \leqslant \operatorname{CVaR}_{\alpha}(Y) \quad \forall \alpha \in[0,1],
$$

see Theorem 2.57 in [8]. If $X \succcurlyeq S S D Y$, then (9) implies that $\rho(X) \leqslant \rho(Y)$ for every $\rho$ in the form (7), so that $Y$ can be viewed as universally riskier than $X$.

A coherent risk measure can be required to conform to certain preferences from agent's past experience, for example, to

(a) Accept assets with rates of return $X_{1}, X_{2}, \ldots, X_{k}$, i.e. $X_{1} \in A_{\rho}, \ldots, X_{k} \in A_{\rho}$ or, equivalently,

$$
\rho\left(X_{1}\right) \leqslant 0, \ldots, \rho\left(X_{k}\right) \leqslant 0,
$$

which can be written as $\mathcal{Q} \subset \mathcal{Q}_{M}$, where $\mathcal{Q}$ is the risk envelope associated with $\rho$ and

$$
\mathcal{Q}^{M}=\left\{Q \in \mathcal{Q}^{M A X} \mid E\left[Q X_{1}\right] \geqslant 0, \ldots, E\left[Q X_{k}\right] \geqslant 0\right\} .
$$

In other words, the agent's risk envelope should include only those scenarios, under which $X_{1}, X_{2}, \ldots, X_{k}$ have non-negative expected values.

(b) Order "reference" rates $X_{1}, \ldots, X_{k}$ according to a given set $S$ of orderings: $(i, j) \in S \Leftrightarrow X_{i} \succeq X_{j}$. For example, the agent may feel that the FTSE250 index is safer than the FTSE100 index, which, in turn, is safer than the IBM stock. Such preferences may be formalized as $\mathcal{Q} \subset \mathcal{Q}_{M}$, where $\mathcal{Q}$ is the risk envelope of $\rho$ and

$$
\mathcal{Q}^{M}=\left\{Q \in \mathcal{Q}^{M A X} \mid E\left[Q X_{i}\right] \geqslant E\left[Q X_{j}\right],(i, j) \in S\right\} .
$$

(c) Exclude certain (Radon-Nikodym derivatives of) probability measures that are "too concentrated" to some scenarios, e.g. to have $\mathcal{Q} \subset \mathcal{Q}_{M}$ with

$$
\mathcal{Q}^{M}=\left\{Q \in \mathcal{Q}^{M A X} \mid 1 / 2 \leqslant Q \leqslant 3 / 2\right\} .
$$

(d) Generate a risk envelope by perturbing a "reference" element $Q^{*} \in \mathcal{Q}^{M A X}$, e.g. $Q^{*}=1$ :

$$
\mathcal{Q}=\left\{Q \in \mathcal{Q}^{M A X} \mid Q=Q^{*}+Z, Z \in \mathcal{Z}\right\},
$$

where $\mathcal{Z} \subset \mathcal{L}^{1}(\Omega)$ is a closed convex set of possible "errors" of $Q^{*}$, and $Q=Q^{*}+Z$ can be viewed as a perturbation of $Q^{*}$. For example, $\mathcal{Z}$ may consist of all r.v.'s $Z$ with $E[|Z|] \leqslant \epsilon$ for some $\epsilon>0$.

In addition, $\rho$ can be assumed to be either (i) law-invariant, (ii) mixed CVaR (5), or (iii) a finite combination of CVaRs

$$
\rho(X)=\sum_{j=1}^{m} \lambda_{j} \operatorname{CVaR}_{\alpha_{j}}(X)
$$

for some $0 \leqslant \alpha_{1}<\cdots<\alpha_{m} \leqslant 1$ and $\lambda_{j} \geqslant 0, j=1, \ldots, m$ with $\sum_{j=1}^{m} \lambda_{j}=1$.

The agent may combine any requirements (a)-(d) on the existing preferences with any assumption (i)-(iii) on the form of coherent risk measure. In general, let $\mathcal{R}$ be the set of all coherent risk measures satisfying agent's requirements/assumptions. For example, $\mathcal{R}$ can be a set of all law-invariant coherent risk measures such that

$$
\mathcal{R}=\left\{\rho(X)=\sup _{Q \in \mathcal{Q}} E[Q(-X)] \mid \mathcal{Q} \subset \mathcal{Q}^{M}\right\}
$$

with $\mathcal{Q}^{M}$ defined by either (10), (11), or (12), if the agent requires $\rho$ to conform to preference conditions (a), (b), or (c), respectively. 
If $\mathcal{R}$ is not a singleton, how to choose a particular risk measure $\rho \in \mathcal{R}$ ? One of the approaches is to recover $\rho$ from the inverse portfolio problem: the agent assumes that the rate of return of the portfolio that he/she either believes to be optimal or is relatively satisfied with minimizes $\rho$ over the set $\mathcal{X}$. The portfolio optimality conditions are then used to find the risk envelope associated with $\rho$, and $\rho$ is given by (3).

Once $\rho$ is recovered, the agent may use it to find an optimal portfolio over a different set $\mathcal{X}$ of portfolio rates of return, $X$, and subject to additional constraints, e.g. $E[X] \geqslant \pi$ for some $\pi>0$; see e.g. [23]. However, the knowledge of $\rho$ is critical far beyond portfolio selection. One example is optimal risk sharing between two agents who use law-invariant coherent risk measures $\rho_{1}$ and $\rho_{2}$ as numerical representations of their attitudes towards risk. Namely, given agents' endowments $X_{1}$ and $X_{2}$, it is often possible to find agents' shares $Y_{1}$ and $Y_{2}$ of the aggregate endowment $X=X_{1}+X_{2}$ such that $Y_{1}+Y_{2}=X$, but $\rho_{1}\left(Y_{1}\right)<\rho_{1}\left(X_{1}\right)$ and $\rho_{2}\left(Y_{2}\right)<\rho_{2}\left(X_{2}\right)$, see [14]. Another example is hedging in an incomplete market, when, in contrast to a complete market, not every contingent claim can be replicated with the existing set of assets, and the agent needs to find a hedging strategy that minimizes the risk (measured by $\rho$ ) of the residual of a replicated claim, see [32].

\section{Inverse Portfolio Problem}

\subsection{Problem Formulation}

A single-period portfolio optimization problem is formulated as follows. Let r.v.'s $r_{1}, \ldots, r_{n}$ be the rates of return of $n$ risky assets, and let $v_{i}$ be the fraction of the initial capital invested into asset $i$. Then the portfolio rate of return is determined by $r_{p}=\sum_{i=1}^{n} r_{i} v_{i}$, and the investor's portfolio problem is to minimize a coherent risk measure $\rho$ of $r_{p}$ with respect to $v_{1}, \ldots, v_{n}$ subject to the budget constraint $\sum_{i=1}^{n} v_{i}=1$ and no-shorting constraints $v_{i} \geqslant 0, i=1, \ldots, n$ :

$$
\min _{X \in \mathcal{X}} \rho(X),
$$

where $\mathcal{X}$ is the set of all possible rates of return:

$$
\mathcal{X}=\left\{X=\sum_{i=1}^{n} r_{i} v_{i} \mid \sum_{i=1}^{n} v_{i}=1, v_{1} \geqslant 0, \ldots, v_{n} \geqslant 0\right\} .
$$

If short sales are allowed, the feasible set $\mathcal{X}$ in (17) becomes $\mathcal{X}=\left\{X=\sum_{i=1}^{n} r_{i} v_{i} \mid \sum_{i=1}^{n} v_{i}=1\right\}$, and if there is also a risk-free asset with constant rate of return $r_{0}$, then $\mathcal{X}=\left\{X=\sum_{i=0}^{n} r_{i} v_{i} \mid \sum_{i=0}^{n} v_{i}=1\right\}$. In the last case, $v_{0}$ is typically expressed from the budget constraint as $v_{0}=1-\sum_{i=1}^{n} v_{i}$, and $r_{p}$ takes the form $r_{p}=\sum_{i=1}^{n}\left(r_{i}-r_{0}\right) v_{i}+r_{0}=X+r_{0}$, where $X=r_{p}-r_{0}=\sum_{i=1}^{n}\left(r_{i}-r_{0}\right) v_{i}$ is the portfolio excess rate of return. In this case, $\rho\left(r_{p}\right)=\rho(X)-r_{0}$, and the portfolio optimization problem can be equivalently reformulated as (16) with

$$
\mathcal{X}=\left\{X=\sum_{i=1}^{n}\left(r_{i}-r_{0}\right) v_{i} \mid\left(v_{1}, \ldots, v_{n}\right) \in \mathbb{R}^{n}\right\} .
$$

In either case, the inverse portfolio problem is then formulated by

Find $\rho \in \mathcal{R}$ such that $\rho\left(X^{*}\right) \leqslant \rho(X)$ for each $X \in \mathcal{X}$, where $X^{*} \in \mathcal{X}$ and $\mathcal{X}$ is given by either (17) or (18).

Theorem 5 in [27] implies that a sufficient condition for $X^{*}$ to be optimal in (16), which is also necessary when $\rho$ is finite on $\mathcal{L}^{2}(\Omega)$, is the existence of a risk identifier $Q \in \mathcal{Q}_{\rho}\left(X^{*}\right)$ such that

$$
E\left[\left(X-X^{*}\right) Q\right] \leqslant 0 \quad \forall X \in \mathcal{X}
$$



8.1])

With $\mathcal{X}$ given by (17), $X^{*}=\sum_{i=1}^{n} r_{i} v_{i}^{*}$, and (20) reduces to (see also [30, Theorem 2] and [36, Theorem

$$
\exists Q \in \mathcal{Q}_{\rho}\left(X^{*}\right) \quad \text { such that } \quad E\left[r_{i} Q\right]\left\{\begin{array}{ll}
=\zeta, & v_{i}^{*}>0 \\
\leqslant \zeta, & v_{i}^{*}=0
\end{array} \quad i=1, \ldots, n,\right.
$$

where $\zeta$ is the Lagrange multiplier corresponding to the constraint $\sum_{i=1}^{n} v_{i}=1$, which implies that optimal $\zeta$ is equal to $E\left[X^{*} Q\right]=-\rho\left(X^{*}\right)$. The optimality conditions (21) can be equivalently formulated as

$$
\exists Q \in \mathcal{Q} \quad \text { such that } E\left[r_{i} Q\right] \leqslant-\rho\left(X^{*}\right), \quad i=1, \ldots, n .
$$

Indeed, let $Q \in \mathcal{Q}$ satisfy (22). Then (22) yields $\sum_{i=1}^{n}\left(E\left[r_{i} Q\right]+\rho\left(X^{*}\right)\right) v_{i}^{*} \leqslant 0$, so that $E\left[X^{*} Q\right]+\rho\left(X^{*}\right) \leqslant$ 0 , or $\rho\left(X^{*}\right) \leqslant E\left[Q\left(-X^{*}\right)\right]$. Since $Q \in \mathcal{Q}, \rho\left(X^{*}\right) \geqslant E\left[Q\left(-X^{*}\right)\right]$, and the equality holds. Thus, $Q$ is, in fact, a risk identifier for $X^{*}$, and the equality in (22) holds for every $i$ such that $v_{i}^{*}>0$. Hence, (22) reduces to (21).

If $\mathcal{X}$ is given by (18), then the optimal portfolio rate of return must have zero risk.

Proposition 1 If $X^{*}$ is a solution to (16) with $\mathcal{X}$ given by (18), then $\rho\left(X^{*}\right)=0$. Consequently, any portfolio rate of return from the set $\left\{X \in \mathcal{X} \mid X=\alpha X^{*}, \alpha \geqslant 0\right\}$ is also optimal in (16).

Proof Since $\mathcal{X}$ in (18) is a subspace of $\mathcal{L}^{\infty}(\Omega),(1-\epsilon) X^{*} \in \mathcal{X}$ and $(1+\epsilon) X^{*} \in \mathcal{X}$ for some $\epsilon>0$, and since $X^{*}$ is optimal, $\rho\left(X^{*}\right) \leqslant \min \left\{\rho\left((1-\epsilon) X^{*}\right), \rho\left((1+\epsilon) X^{*}\right)\right\}=\min \left\{(1-\epsilon) \rho\left(X^{*}\right),(1+\epsilon) \rho\left(X^{*}\right)\right\}$, which implies that $\rho\left(X^{*}\right)=0$. By positive homogeneity, $\rho\left(\alpha X^{*}\right)=0$ for any $\alpha \geqslant 0$ such that $\alpha X^{*} \in \mathcal{X}$, and consequently, all these portfolio rates of return are optimal as well.

Proposition 1 implies that $E\left[X^{*} Q\right]=\rho\left(X^{*}\right)=0$ for any $Q \in \mathcal{Q}_{\rho}\left(X^{*}\right)$, and consequently, the optimality condition (20) reduces to $E[X Q] \leqslant 0$ for all $X \in \mathcal{X}$, which is possible only if

$$
E[X Q]=0 \quad \forall X \in \mathcal{X}
$$

or, equivalently, if $E\left[r_{i} Q\right]=r_{0}, i=1, \ldots, n$. Any $Q \in \mathcal{Q}^{M A X}$ satisfying (23) is called the Radon-Nikodym derivative of "risk-neutral probability measure" or "risk neutral" in short. ${ }^{8}$ By the fundamental theorem of asset pricing (see, e.g., [8, Theorem 1.7]), the existence of the risk-neutral probability measure is equivalent to the no-arbitrage assumption, i.e. no portfolio has excess return non-negative with probability 1 and strictly positive with positive probability. If this assumption does not hold, the investor can borrow at the risk-free rate unlimited amount of money and can invest in the risky assets making portfolio risk as small as desired, so that there is no optimal portfolio, and the inverse portfolio problem (19) has no solution.

\subsection{Law-invariant Coherent Risk Measure}

This section seeks to find law-invariant solutions to the inverse portfolio problem (19). The following proposition establishes necessary and sufficient conditions for their existence.

Proposition 2 For arbitrary set $\mathcal{X}$ and any $X^{*} \in \mathcal{X}$, the following conditions are equivalent:

(a) The inverse problem (19) has a solution in the form of worst-case mixed CVaR (7).

(b) There is no constant $\epsilon>0$ and $Y \in \mathcal{X}$ such that $Y \succcurlyeq_{S S D} X^{*}+\epsilon$.

(c) There is no $Y \in \mathcal{X}$ such that

$$
\operatorname{CVaR}_{\alpha}(Y)<\operatorname{CVaR}_{\alpha}\left(X^{*}\right) \quad \forall \alpha \in[0,1] .
$$

\footnotetext{
${ }^{8} \mathrm{~A}$ risk-neutral probability measure is a measure under which the expected discounted future price of any instrument is equal to the current one, see [8].
} 
(d) The inverse problem (19) has a solution in the form of mixed CVaR (5).

If the probability space $\Omega$ is either atomless or finite uniform, i.e. $\Omega=\left\{\omega_{1}, \ldots, \omega_{T}\right\}$ with $\mathbb{P}\left[\omega_{t}\right]=1 / T$, $t=1, \ldots, T$, then conditions $(a)-(d)$ are equivalent to the condition that

(e) The inverse problem (19) has a law-invariant solution.

If the probability space $\Omega$ is finite uniform, then conditions $(a)-(e)$ are equivalent to the condition that

(f) The inverse problem (19) has a solution in the form (14) with $m=T$ and $\alpha_{j}=j / T, j=1, \ldots, T$.

If $\mathcal{X}$ is given by (18), then conditions $(a)-(d)$ are equivalent to the conditions that

(g) There exists a risk-neutral r.v. $Q^{*} \in \mathcal{Q}^{M A X}$ comonotone ${ }^{9}$ with $-X^{*}$.

(h) The inverse problem (19) has a solution in the form

$$
\rho(X)=\int_{0}^{1} q_{Q^{*}}(\alpha) q_{-X}(\alpha) d \alpha, \quad Q^{*} \in \mathcal{Q}^{M A X} .
$$

Proof $(\mathrm{a}) \Rightarrow(\mathrm{b})$ : By contradiction, let $\epsilon>0$ and $Y \in \mathcal{X}$ be such that $Y \succcurlyeq_{S S D} X^{*}+\epsilon$. Then, for any $\rho$ given by (7), $\rho(Y) \leqslant \rho\left(X^{*}+\epsilon\right)=\rho\left(X^{*}\right)-\epsilon<\rho\left(X^{*}\right)$, hence $\rho$ is not a solution to the inverse problem (19).

(b) $\Rightarrow$ (c): By contradiction, let $Y \in \mathcal{X}$ be such that (24) holds. Since $\mathrm{CVaR}_{\alpha}\left(X^{*}\right)-\mathrm{CVaR}_{\alpha}(Y)$ is a continuous function of $\alpha \in[0,1]$, it attaints its minimum value $\epsilon$. By (24), $\epsilon>0$, and $\operatorname{CVaR}_{\alpha}(Y) \leqslant$ $\mathrm{CVaR}_{\alpha}\left(X^{*}\right)-\epsilon=\mathrm{CVaR}_{\alpha}\left(X^{*}+\epsilon\right)$ for every $\alpha \in[0,1]$. Hence, $Y \succcurlyeq_{S S D} X^{*}+\epsilon$, which contradicts (b).

(c) $\Rightarrow$ (d): The convexity of $\mathcal{X}$ and $\mathrm{CVaR}_{\alpha}, \alpha \in[0,1]$, implies that the set $\mathcal{U}=\{\phi \in \mathcal{S} \mid \exists X \in \mathcal{X}: \phi(\alpha) \geqslant$ $\left.\mathrm{CVaR}_{\alpha}(X)\right\}$ is a convex subset in the vector space $\mathcal{S}$ of continuous functions $\phi:[0,1] \rightarrow \mathbb{R}$. By (c), the function $\phi^{*}(\alpha)=\mathrm{CVaR}_{\alpha}\left(X^{*}\right)$ belongs to $\mathcal{U}$, but not to the interior of $\mathcal{U}$. Thus, there exists a non-zero linear functional $\mathcal{L}: \mathcal{S} \rightarrow \mathbb{R}$ which attains its supremum on $\mathcal{U}$ at $\phi^{*}$; see e.g. [37, Corollary 1.1.4]. In fact, $(-\mathcal{L})$ is a positive linear functional, that is, $(-\mathcal{L})(\phi) \geqslant 0$ for any function $\phi$ such that $\phi(\alpha) \geqslant 0$ for all $\alpha \in[0,1]$. Indeed, $\phi^{*}+\phi \in \mathcal{U}$, hence $\mathcal{L}\left(\phi^{*}+\phi\right) \leq \mathcal{L}\left(\phi^{*}\right)$, so that $\mathcal{L}(\phi) \leqslant 0$. Thus, $(-\mathcal{L})(\phi)=\int_{0}^{1} \phi(\alpha) d \lambda(\alpha)$ for some non-negative measure $\lambda$ on $[0,1]$, and we may assume that $\int_{0}^{1} d \lambda(\alpha)=1$. Then the functional $\mathcal{R}(X)=-\mathcal{L}\left(\mathrm{CVaR}_{\alpha}(X)\right)=\int_{0}^{1} \operatorname{CVaR}_{\alpha}(X) d \lambda(\alpha)$ belongs to the family (5) and attains its infimum on $\mathcal{X}$ at $X^{*}$.

(a) $\Rightarrow$ (f): Let $\widetilde{\rho}$ be a solution to the inverse problem in the form (7). By Theorem 5 in [27], there exists a risk identifier $Q^{*} \in \mathcal{Q}_{\widetilde{\rho}}\left(X^{*}\right)$ such that $E\left[\left(X-X^{*}\right) Q^{*}\right] \leqslant 0$ for all $X \in \mathcal{X}$. By Proposition $1, \widetilde{\rho}\left(X^{*}\right)=0$. Since $Q^{*} \in \mathcal{Q}_{\widetilde{\rho}}\left(X^{*}\right)$, this implies that $E\left[X^{*} Q^{*}\right]=0$, and consequently, $E\left[X Q^{*}\right] \leqslant 0$ for all $X \in \mathcal{X}$, which is possible only if $E\left[Q^{*} r_{i}\right]=r_{0}, i=1, \ldots, n$. Thus, $Q^{*}$ is a risk-neutral r.v. If $\rho(X) \not \equiv-E[X]$, then $Q^{*}$ is comonotone with $-X^{*}$ by Proposition 14 in [12], applied to the functional $\mathcal{D}(X)=\rho(X)+E[X]$. If $\rho(X) \equiv-E[X]$ then $Q^{*} \equiv 1$, and comonotonicity follows as well.

$(\mathrm{f}) \Rightarrow(\mathrm{h})$ : Let $Q^{*} \in \mathcal{Q}^{M A X}$ be a risk-neutral r.v. comonotone with $-X^{*}$. Then

$$
\rho\left(X^{*}\right)=\int_{0}^{1} q_{Q^{*}}(\alpha) q_{-X^{*}}(\alpha) d \alpha=E\left[Q^{*}\left(-X^{*}\right)\right]=0,
$$

where the second equality follows from comonotonicity of $Q^{*}$ and $-X^{*}$, and the third one from risk neutrality of $Q^{*}$. On the other hand,

$$
\rho(X)=\int_{0}^{1} q_{Q^{*}}(\alpha) q_{-X}(\alpha) d \alpha \geqslant E\left[Q^{*}(-X)\right]=0=\rho\left(X^{*}\right)
$$

\footnotetext{
${ }^{9}$ Two r.v.'s $X: \Omega \rightarrow \mathbb{R}$ and $Y: \Omega \rightarrow \mathbb{R}$ are said to be comonotone, if there exists a set $A \subset \Omega$ such that $P[A]=1$ and $\left(X\left(\omega_{1}\right)-X\left(\omega_{2}\right)\right)\left(Y\left(\omega_{1}\right)-Y\left(\omega_{2}\right)\right) \geqslant 0$ for all $\omega_{1}, \omega_{2} \in A$.
} 
for any other r.v. $X \in \mathcal{X}$, where the inequality is due to Hardy \& Littlewood [8, Theorem A.24]. Hence, $X^{*}$ is optimal in (16) with $\rho$.

Implications (h) $\Rightarrow$ (a) and (d) $\Rightarrow$ (a) follow from the fact that families (25) and (5) are subclasses of (7). On an atomless probability space, every law-invariant risk measure can be represented in the form (7) [8, Corollary $4.63])$, and (a) $\Leftrightarrow$ (e) follows.

Let $\Omega$ be a finite uniform set with $T$ elements. Then every law-invariant risk measure $\rho$ preserves SSD, i.e. $\rho(X) \leqslant \rho(Y)$ whenever $X \succcurlyeq S S D Y$, see [4, Theorem 3.5], so that it can be represented in the form (7) [19, Theorem 2.2], and (a) $\Leftrightarrow$ (e) follows. Finally, with finite uniform $\Omega, \mathrm{CVaR}_{\alpha}(X)$ is a piecewise-linear function of $\alpha$ for every $X$, so that the condition (24) can be written as

$$
\mathrm{CVaR}_{j / T}(Y)<\mathrm{CVaR}_{j / T}\left(X^{*}\right) \quad j=1, \ldots, T,
$$

and (c) $\Rightarrow$ (e) follows from the same argument as (c) $\Rightarrow(d)$, while the implication (e) $\Rightarrow(d)$ is obvious.

If in the proof of (a) $\Leftrightarrow(\mathrm{f}) \Leftrightarrow(\mathrm{h})$ in Proposition $2, \mathcal{Q}^{M A X}$ is replaced by any law-invariant $\mathcal{Q}^{M} \subset \mathcal{Q}^{M A X}$, then the following result holds.

Proposition 3 The inverse problem (19), where $\mathcal{X}$ is given by (18) and $\mathcal{R}$ is given by (15) with law-invariant $\mathcal{Q}^{M}$, has a solution in the form (7) if and only if there exists a risk-neutral r.v. $Q^{*} \in \mathcal{Q}^{M}$ comonotone with $-X^{*}$. In this case, the coherent risk measure

$$
\rho(X)=\int_{0}^{1} q_{Q^{*}}(\alpha) q_{-X}(\alpha) d \alpha
$$

is a solution of the inverse problem.

The solution to the inverse problem (19), if exists, may in general be not unique. Let $\mathcal{R}^{*}$ be the set of all solutions in the form (8) to the inverse problem (19) with $\mathcal{R}$ given by (15).

Proposition 4 If the set $\mathcal{R}^{*}$ is non-empty, it contains a solution $\rho^{*}$ with the associated risk envelope $\mathcal{Q}^{*}$, which is "maximal" possible in sense that $\mathcal{Q} \subset \mathcal{Q}^{*}$ for any other solution $\rho \in \mathcal{R}^{*}$ with the associated risk envelope Q. Moreover, $\mathcal{Q}^{*}$ is given by

$$
\mathcal{Q}^{*}=\left\{Q \in \mathcal{Q}^{M} \mid \int_{0}^{1} q_{Q}(\alpha) q_{-X^{*}}(\alpha) d \alpha \leqslant 0\right\} .
$$

Proof Let $\rho$ given by (8) with the associated risk envelope $\mathcal{Q}$ be a solution to the inverse portfolio problem (19), and let $Q \in \mathcal{Q}$. Then

$$
0=\rho\left(X^{*}\right) \geqslant \int_{0}^{1} q_{Q}(\alpha) q-X^{*}(\alpha) d \alpha,
$$

where the equality follows from Proposition 1, and the inequality is due to (8). Hence, $Q \in \mathcal{Q}^{*}$ by (27), and $\mathcal{Q} \subset \mathcal{Q}^{*}$, and consequently, $\rho^{*}(X) \geqslant \rho(X) \geqslant 0$ for any r.v. $X \in \mathcal{X}$. On the other hand, $\rho^{*}\left(X^{*}\right)=0$ by construction, so that $X^{*}$ is optimal in (16) with $\rho^{*}$.

In the portfolio problem (16), the investor may impose a constraint on the portfolio expected rate of return:

$$
\min _{X \in \mathcal{X}} \rho(X) \text { subject to } E[X] \geqslant \pi \text {. }
$$

The problem inverse to (28) is formulated similarly to (19): given $X^{*} \in \mathcal{X}$, find $\pi$ and $\rho$ such that $X^{*}$ is optimal in (28). If $\rho$ is an averse measure of risk, i.e. $\rho(X)>-E[X]$ for all non-constant $X$ (see [27, 28] and [36, Section 2.3]), and the constraint $E[X] \geqslant \pi$ holds as equality at optimality, then (28) is equivalent to

$$
\min _{X \in \mathcal{X}} \mathcal{D}(X) \quad \text { subject to } E[X] \geqslant \pi
$$

where $\mathcal{D}(X)=\rho(X)+E[X]$ is a deviation measure (see [27,28] and [36, Section 2.1]). The problem inverse to (29) is solved in [12]. 
Proposition 5 [Proposition 4 in [12]] If there is no $Y \in \mathcal{X}$ for which (24) holds, then $X^{*} \in \mathcal{X}$ is an optimal solution in (28) for $\pi=E\left[X^{*}\right]$ and for a risk measure $\rho$ in the form of mixed CVaR (5).

Example 1 Let $\Omega=\left\{\omega_{1}, \ldots, \omega_{T}\right\}$ with $\mathbb{P}\left[\omega_{t}\right]=1 / T, t=1, \ldots, T$, and let $r_{i t}, i=1, \ldots, n, t=1, \ldots, T$, be the excess rate of return of risky asset $i$ under $\omega_{t}$. The feasible set $\mathcal{X}$ in (18) is then a subset of $\mathbb{R}^{T}$ and is given by

$$
\mathcal{X}=\left\{X=\left(x_{1}, \ldots, x_{T}\right) \mid x_{t}=\sum_{i=1}^{n} r_{i t} v_{i}, \quad t=1, \ldots, T, \quad\left(v_{1}, \ldots, v_{n}\right) \in \mathbb{R}^{n}\right\} .
$$

Fix $X^{*}=\left(x_{1}^{*}, \ldots, x_{T}^{*}\right) \in \mathcal{X}$. If there exists a law-invariant solution to the inverse problem (19) with $\mathcal{R}$ given by (15) with $\mathcal{Q}^{M}=\mathcal{Q}^{M A X}$, then its risk envelope (27) takes the form

$$
\mathcal{Q}^{*}=\left\{Q=\operatorname{Perm}\left(q_{1}, \ldots, q_{T}\right) \mid \sum_{t=1}^{T} q_{t}\left(-\bar{x}_{t}^{*}\right) \leqslant 0, \quad \sum_{t=1}^{T} q_{t}=T, \quad 0 \leqslant q_{1} \leqslant q_{2} \leqslant \cdots \leqslant q_{T}\right\},
$$

where $\left(\bar{x}_{1}^{*}, \ldots, \bar{x}_{T}^{*}\right)$ is a permutation of $\left(x_{1}^{*}, \ldots, x_{T}^{*}\right)$ such that $\bar{x}_{1}^{*} \geqslant \cdots \geqslant \bar{x}_{T}^{*}$, and Perm $\left(q_{1}, \ldots, q_{T}\right)$ denotes the set of all permutations of $\left(q_{1}, \ldots, q_{T}\right)$.

In particular, we select $n=92$ instruments from the FTSE 100 index ${ }^{10}$ and identify $T=182$ scenarios for instruments' weekly rates of return from 3-January-2011 to 30-June-2014. Here, $r_{0}=0$, and $r_{i t}$ is the historical rate of return of instrument $i$ during time period $t$.

(i) Let $X^{*}=\left(x_{1}^{*}, \ldots, x_{T}^{*}\right)$ be the rate of return of the master fund of positive type [29] ${ }^{11}$ in Markowitz's mean-variance optimization problem. Figure 1 depicts three elements from the law-invariant risk envelope (30). The horizontal axis denotes scenarios $t=1, \ldots, T=182$, while the vertical one denotes the values of the corresponding $q_{t}-s$.

(ii) Let $X^{*}=\left(x_{1}^{*}, \ldots, x_{T}^{*}\right)$ be the rate of return of the FTSE 100 index. ${ }^{12}$ Then condition $(b)$ in Proposition 2 does not hold, hence there are no law-invariant solution to the inverse problem (19).

There are two reasons for presenting Example 1(i), where $X^{*}$ is chosen to be the rate of return of a meanvariance portfolio. Firstly, Markowitz's mean-variance portfolio is often viewed as a reference result in the modern portfolio theory and capital asset pricing. However, the standard deviation (or, equivalently, variance) is not, in fact, a measure of risk but rather the one of deviation. It does not preserve monotonicity of a preference relation and, as a deviation, cannot be used alone to express risk aversion. In this case, the question of interest is not whether the Markowitz's mean-variance portfolio is desirable, but rather what a "rational equivalent" of the mean-variance model in the class of risk measures, which are monotone and risk averse, is. In other words, the inverse portfolio problem allows to uncover the "rational side" of the mean-variance model. Secondly, Example 1(ii) shows that for $X^{*}$ of a real-life instrument, e.g. the FTSE 100 index, the inverse problem typically may not have exact law-invariant solution. In this case, the investor has two options:

(a) Solve the inverse problem to find an exact non law-invariant solution, or

(b) Find a law-invariant risk measure, which solves the inverse problem approximately.

Option (a) is addressed in Section 3.3, whereas option (b) is pursued in Sections 4.1-4.4.

\footnotetext{
${ }^{10}$ There are 101 instrument in the index, nine of them are excluded due to the lack of data.

${ }^{11} \mathrm{~A}$ master fund of positive/negative type is an optimal mean-deviation portfolio that has positive/negative unit price and zero weight in the risk-free asset, see [29, Definition 2, p. 758]. It is a "tangent portfolio" on the mean-deviation positive/negative efficient frontier of risky assets.

${ }^{12}$ The weights of components in FTSE 100 index can be found at http://www.ftse.com/analytics/factsheets/Home/ConstituentsWeights. The weights of nine excluded components are assumed to be zero.
} 


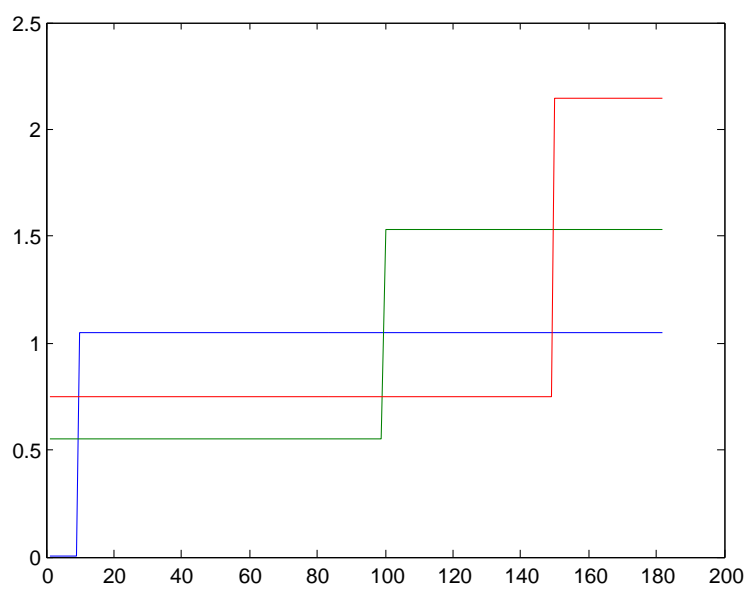

Figure 1: Three elements from the law-invariant risk envelope (30). The horizontal axis denotes scenarios $t=1, \ldots, T=182$, whereas the vertical one denotes the values of the corresponding $q_{t}$-s.

\subsection{General Solution}

If the inverse problem (19) with $\mathcal{X}$ given by (18) has no law-invariant solution, it can always be solved in the class of all coherent measures provided that the market is arbitrage-free. ${ }^{13}$ Indeed, if one takes $\mathcal{Q}=\left\{Q^{*}\right\}$ for any risk neutral $Q^{*}$, then the corresponding coherent risk measure $\rho$ in (3) satisfies $\rho(X)=0$ for all $X \in \mathcal{X}$, so that any $X \in \mathcal{X}$ is optimal, in particular, a given $X^{*} \in \mathcal{X}$. However, there exists $\rho$ for which $X^{*}$ is optimal, but any $X \in \mathcal{X}$, which is not proportional to $X^{*}$, is not. Such $\rho$ can be constructed through (13): start with any risk neutral $Q^{*}$ and then "robustify" it by choosing an appropriate error set $\mathcal{Z}$. Then $Q \in \mathcal{Q}^{M A X}$ for $Q=Q^{*}+Z$ implies that

$$
E[Z]=0 \quad \forall Z \in \mathcal{Z}
$$

Suppose that $Q^{*}$ is a risk identifier for $X^{*}$, then $E\left[Q^{*}\left(-X^{*}\right)\right] \geqslant E\left[\left(Q^{*}+Z\right)\left(-X^{*}\right)\right]$ for all $Z \in \mathcal{Z}$, or, equivalently,

$$
E\left[Z X^{*}\right] \geqslant 0 \quad \forall Z \in \mathcal{Z}
$$

Proposition 6 Let $\mathcal{Q}$ be defined by (13), where $Q^{*}$ is any risk neutral r.v. such that $\mathbb{P}\left[Q^{*}>0\right]=1$, and let $\mathcal{Z}$ be the set of all r.v.'s in $\mathcal{L}^{1}(\Omega)$ satisfying the conditions (31) and (32), ${ }^{14}$ i.e. $\mathcal{Z}=\left\{Z \in \mathcal{L}^{1}(\Omega) \mid E[Z]=\right.$ $\left.0, E\left[Z X^{*}\right] \geqslant 0\right\}$. Then for the corresponding coherent risk measure $\rho$ in (3), the set of optimal portfolio rates of return in (16) is precisely the set $\left\{X \in \mathcal{X} \mid X=\alpha X^{*}, \alpha \geqslant 0\right\}$.

Proof $E\left[Q\left(-X^{*}\right)\right]=E\left[\left(Q^{*}+Z\right)\left(-X^{*}\right)\right]=E\left[Q\left(-X^{*}\right)\right]+E\left[Z\left(-X^{*}\right)\right] \leqslant 0$ for every $Q \in \mathcal{Q}$, where the last inequality follows from (23) and (32). Since the equality holds for, say, $Q=Q^{*}$, we have $\rho\left(X^{*}\right)=0$.

For any $X \in \mathcal{X}$, let $\mathcal{Z}_{X} \in \mathcal{L}_{1}(\Omega)$ be a set of r.v.'s $Z$ such that $E[Z]=0$ and $E[Z X] \geqslant 0$. If $X=\alpha X^{*}$ for some $\alpha \geqslant 0$, then $\mathcal{Z}_{X}=\mathcal{Z}$, but otherwise $\mathcal{Z} \backslash \mathcal{Z}_{X}$ is non-empty, and there is an r.v. $Z^{*}$ such that $E\left[Z^{*}\right]=0$ and $E\left[Z^{*} X^{*}\right]>0>E\left[Z^{*} X\right]$. Since bounded r.v.'s are dense in $\mathcal{L}^{1}(\Omega), Z^{*}$ can be chosen to be bounded. For any $\epsilon>0$, let $Z_{\epsilon}$ be an r.v. such that $Z_{\epsilon}(\omega)=0, \omega \in \Omega_{\epsilon}$ and $Z_{\epsilon}(\omega)=Z^{*}+C_{\epsilon}, \omega \notin \Omega_{\epsilon}$, where $\Omega_{\epsilon}=\left\{\omega \mid Q^{*}(\omega) \leqslant \epsilon\right\}$ and $C_{\epsilon}$ is chosen such that $E\left[Z_{\epsilon}\right]=0$. Since $\mathbb{P}\left[Q^{*}>0\right]=1, \lim _{\epsilon \rightarrow 0} \mathbb{P}\left[\Omega_{\epsilon}\right]=0$, so that $\lim _{\epsilon \rightarrow 0} Z_{\epsilon}=Z^{*}$ in $\mathcal{L}^{1}(\Omega)$. Thus, $E\left[Z_{\epsilon} X^{*}\right]>0>E\left[Z_{\epsilon} X\right]$ for sufficiently small $\epsilon$. Then $E[Q]=1$ and $Q \geqslant 0$

\footnotetext{
${ }^{13} \mathrm{~A}$ market is considered to be the set of one risk-free and $n$ risky financial instruments, whereas an arbitrage-free market is the one in which no portfolio has excess rate of return non-negative with probability 1 and strictly positive with positive probability; see [8].

${ }^{14}$ The statement of the proposition will be unaffected if the extra condition that $\|Z\|_{1} \leqslant \epsilon$ for all $Z \in \mathcal{Z}$ and some $\epsilon>0$ is added.
} 
for $Q=Q^{*}+\frac{\epsilon}{\left\|Z_{\epsilon}\right\|_{\infty}} Z_{\epsilon}$. Consequently, $Q \in \mathcal{Q}$ and $\rho(X) \geqslant E[Q(-X)]=E\left[\left(Q^{*}+\frac{\epsilon}{\left\|Z_{\epsilon}\right\|_{\infty}} Z_{\epsilon}\right)(-X)\right]=$ $-\frac{\epsilon}{\left\|Z_{\epsilon}\right\|_{\infty}} E\left[Z_{\epsilon} X\right]>0$, and the statement is proved.

Under the conditions in Proposition 1, Proposition 6 describes the best possible solution for the inverse portfolio problem (19). In terms of acceptance sets, it returns the coherent risk measure $\rho$, for which the given rate of return $X^{*}$ along with $\alpha X^{*}$ for any $\alpha>0$ is acceptable, but any other r.v. $X$ is not.

In general, the set $\mathcal{Q}$ in Proposition 6 is not unique: if the risk neutral $Q^{*}$ is not unique, the investor can choose either a "favorite" or the maximal possible set $\mathcal{Q}$ such that the corresponding coherent risk measure is a solution to the inverse portfolio problem (19).

Proposition 7 The inverse problem (19) with $\mathcal{X}$ given by (18) and with $\mathcal{R}$ given by (15) has a solution if and only if there exists a risk-neutral r.v. $Q^{*} \in \mathcal{Q}^{M}$. In this case, a solution $\rho$ is determined by (3) with

$$
\mathcal{Q}=\left\{Q \in \mathcal{Q}^{M} \mid E\left[Q X^{*}\right] \geqslant 0\right\}
$$

and this solution is "maximal" possible in sense that the risk envelope of any other solution is a subset of $\mathcal{Q}$ in (33).

Proof "Only if": Let $\widetilde{\rho} \in \mathcal{R}$ be a solution to (19). By Proposition 1, $\widetilde{\rho}\left(X^{*}\right)=0$, and consequently, $\widetilde{\rho}(X) \geqslant 0$ for all $X \in \mathcal{X}$. By Theorem 5 in [27], there exists a risk identifier $Q^{*} \in \mathcal{Q}_{\widetilde{\rho}}\left(X^{*}\right)$ such that $E\left[\left(X-X^{*}\right) Q^{*}\right] \leqslant 0$ for all $X \in \mathcal{X}$. Hence, $E\left[X Q^{*}\right] \leqslant E\left[X^{*} Q^{*}\right]=\widetilde{\rho}\left(X^{*}\right)=0$ for all $X \in \mathcal{X}$. Since $\mathcal{X} \subseteq \mathcal{L}^{\infty}(\Omega)$, this implies that $Q^{*}$ is a risk-neutral r.v. Since $\widetilde{\rho} \in \mathcal{R}$ and since $Q^{*}$ is in the risk envelope of $\widetilde{\rho}$, it follows from (15) that $Q^{*} \in \mathcal{Q}^{M}$.

"If": Let $Q^{*} \in \mathcal{Q}^{M}$ be risk-neutral r.v. Then $Q^{*} \in \mathcal{Q}$ by (33), so that $\rho(X) \geqslant E\left[Q^{*}(-X)\right]=0$ for all $X \in \mathcal{X}$. Since $\rho\left(X^{*}\right) \leqslant 0$ by construction, $\rho$ is a solution to (19). If $\widetilde{\rho} \in \mathcal{R}$ is any other solution with a corresponding risk envelope $\mathcal{Q}^{\prime}, \widetilde{\rho}\left(X^{*}\right)=0$ by Proposition 1 , and consequently, $E\left[Q X^{*}\right] \geqslant 0$ for every $Q \in \mathcal{Q}^{\prime}$. Since $\mathcal{Q}^{\prime} \subset \mathcal{Q}^{M}$ by (15), this implies that $\mathcal{Q}^{\prime} \subset \mathcal{Q}$.

The solution (33) can be viewed as a robust approach to identifying risk preferences: it includes the RadonNikodym derivatives of all possible scenarios, for which given rate of return $X^{*}$ belongs to investor's acceptance set, i.e. $X^{*}$ is not worse than the risk-free rate. Observe that (33) is independent of the feasible set $\mathcal{X}$.

Example 2 In the settings of Example 1, the risk envelope (33) of a solution with $\mathcal{Q}^{M}=\mathcal{Q}^{M A X}$ to the inverse problem (19) is given by

$$
\mathcal{Q}=\left\{Q=\left(q_{1}, \ldots, q_{T}\right) \mid \sum_{t=1}^{T} q_{t} x_{t}^{*} \geqslant 0, \quad \sum_{t=1}^{T} q_{t}=T, \quad q_{1} \geqslant 0, \ldots, q_{T} \geqslant 0\right\}
$$

If $\mathcal{Q}^{M}$ is given by (10), the inverse problem (19) can be formulated as follows: find a coherent risk measure $\rho$ for which $X^{*}$ is optimal, provided that $X_{1}, \ldots, X_{k}$ are acceptable. In this case, the solution (33) includes additional constraints $E\left[Q X_{i}\right] \geqslant 0, i=1, \ldots, k$ :

$$
\mathcal{Q}=\left\{Q \in \mathcal{Q}^{M A X} \mid E\left[Q X^{*}\right] \geqslant 0, E\left[Q X_{1}\right] \geqslant 0, \ldots, E\left[Q X_{k}\right] \geqslant 0\right\},
$$

which, obviously, remains the same if $X^{*}$ is interchanged with any of $X_{1}, \ldots, X_{k}$.

The representation (33) can be readily generalized for the case when the rate of return of investor's multiperiod portfolio is known. Suppose that at each time moment $t \in \mathcal{T}=\{0,1,2,3, \ldots\}$, the investor chooses a portfolio, optimal for the next time period, i.e. the investor solves a single-period optimization problem

$$
\min _{X_{t+1} \in \mathcal{X}_{t}} \rho\left(X_{t+1}\right)
$$


where $X_{t+1}$ is the excess rate of return of the portfolio at time $t+1$ subject to the information available at time $t$, and $\mathcal{X}_{t}$ is the set of all possible excess rates of return available at time $t$.

The problem inverse to (34) is then formulated as follows. Let $X^{*}(t)$ be the excess rate of return of the optimal portfolio at $t \in \mathcal{T}$. If $X^{*}(t)$ is known for each $t \in \mathcal{T}$, find $\rho$ such that $X^{*}(t)$ is optimal in (34) for each $t \in \mathcal{T}$. The requirement of the optimality of $X^{*}(t)$ can be replaced by a weaker condition that for each $t \in \mathcal{T}$, $X^{*}(t)$ belongs to the investor's acceptance set. Then the investor's risk envelope can be recovered as

$$
\mathcal{Q}=\left\{Q \in \mathcal{Q}^{M} \mid E\left[Q X^{*}(t)\right] \geqslant 0 \forall t \in \mathcal{T}\right\}
$$

where $\mathcal{Q}^{M} \subset \mathcal{Q}^{M A X}$ is specified by extra constraints that the investor may wish to add.

If the set (35) is empty, then investor's risk preferences are incompatible with coherent risk measures and the investor may opt for an approximate solution by replacing 0 in the right-hand in (35) side by some $\epsilon<0$.

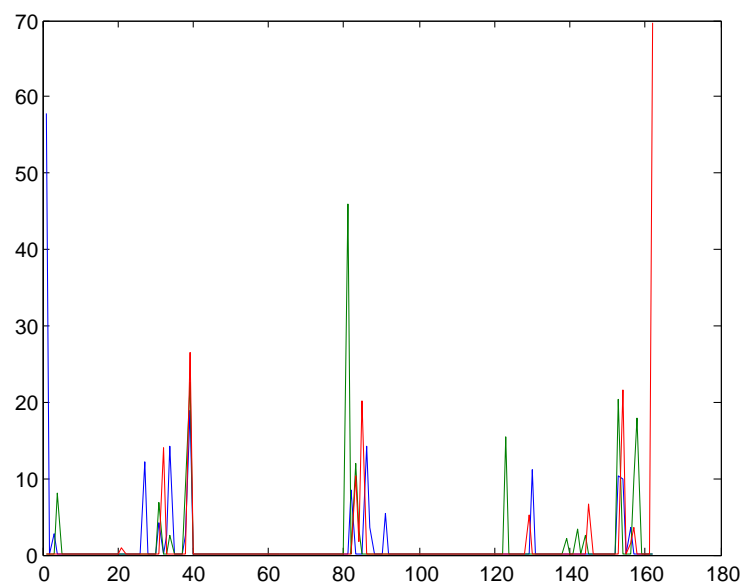

(a) unsorted $q_{t}-\mathrm{s}$

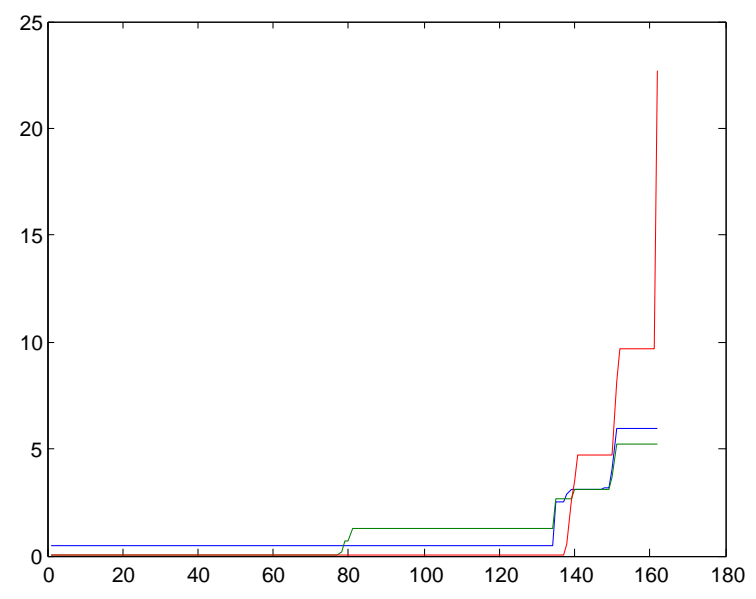

(b) sorted $q_{t}-\mathrm{s}$

Figure 2: A solution of the inverse multi-period portfolio problem (36). The horizontal axis denotes scenarios $t=1, \ldots, T$ with $T=162$, whereas the vertical one denotes the values of the corresponding $q_{t}$-s.

Example 3 In the settings of Example $1(i)$, let $X^{t}=\left(x_{1}^{t}, \ldots, x_{T}^{t}\right)$ be the excess rate of return of the portfolio that the investor held at times $t=1,2, \ldots, t_{0}$. Then the investor's risk envelope (35) with $\mathcal{Q}^{M}=\mathcal{Q}^{M A X}$ is given by

$$
\mathcal{Q}=\left\{Q=\left(q_{1}, \ldots, q_{T}\right) \mid \sum_{j=1}^{T} q_{j} x_{j}^{t} \geqslant 0, \quad t=1, \ldots, t_{0}, \quad \sum_{j=1}^{T} q_{j}=T, \quad q_{1} \geqslant 0, \ldots, q_{T} \geqslant 0\right\} .
$$

Suppose that the investor holds a portfolio with the rate of return $X^{*}$ for the last $t_{0}=20$ weeks. We use (36) with $t_{0}=20, T=182-20=162$, and $x_{j}^{t}=x_{j+t-1}^{*}, j=1, \ldots, T, t=1, \ldots, t_{0}$ to determine the risk envelope $\mathcal{Q}$. Figure 2 a shows three random elements from $\mathcal{Q}$. Figure $2 b$ shows three elements from $\mathcal{Q}$ when additional constraints $0 \leqslant q_{1} \leqslant q_{2} \leqslant \cdots \leqslant q_{T}$, giving more weight to more recent observations, are added to (36). The horizontal axis denotes scenarios $j=1, \ldots, T$, while the vertical one denotes the values of the corresponding $q_{j}-s$.

\section{Inverse Portfolio Problem with mixed CVaR}

Suppose that a solution $\rho$ to the inverse problem (19) is sought in the form of mixed CVaR (14). If the underlying probability space $\Omega$ is finite uniform, which is typically the case in the scenario-based apparoch, such a solution 
always exists, provided that there exists a law-invariant solution, see Proposition 2 . We assume that $\alpha_{1}, \ldots, \alpha_{m}$ in (14) are fixed, so that the problem reduces to finding $\lambda_{1}, \ldots, \lambda_{m}$.

In this case, any element $Q$ of the risk envelope $\mathcal{Q}$ can be represented by

$$
Q=\sum_{j=1}^{m} \lambda_{j} Q_{j}
$$

where $Q_{j}$ belongs to the risk envelope $\mathcal{Q}_{j}$ of $\mathrm{CVaR}_{\alpha_{j}}$ given by

$$
\mathcal{Q}_{j}=\left\{Q \in \mathcal{L}^{1}(\Omega) \mid E[Q]=1, Q \in\left[0,1 / \alpha_{j}\right]\right\}, j=1, \ldots, m
$$

Then the optimality conditions (22) can be written as

$$
\begin{aligned}
\exists Q_{1}, \ldots, Q_{m} \quad \text { such that } \quad & E\left[Q_{j}\right]=1, \quad Q_{j} \in\left[0,1 / \alpha_{j}\right], \quad j=1, \ldots, m, \\
& \sum_{j=1}^{m} E\left[r_{i} \lambda_{j} Q_{j}\right] \leqslant-\sum_{j=1}^{m} \lambda_{j} \operatorname{CVaR}_{\alpha_{j}}\left(X^{*}\right), \quad i=1, \ldots, n,
\end{aligned}
$$

which, with new variables $S_{1}=\lambda_{1} Q_{1}, \ldots, S_{m}=\lambda_{m} Q_{m}$, simplifies to a linear system of equations and inequalities for $\lambda_{1}, \ldots, \lambda_{m}, S_{1}, \ldots, S_{m}$ :

$$
\begin{aligned}
& \sum_{j=1}^{m} E\left[r_{i} S_{j}\right] \leqslant-\sum_{j=1}^{m} \lambda_{j} \operatorname{CVaR}_{\alpha_{j}}\left(X^{*}\right), \quad i=1, \ldots, n, \\
& E\left[S_{j}\right]=\lambda_{j}, \quad S_{j} \in\left[0, \lambda_{j} / \alpha_{j}\right], \quad j=1, \ldots, m, \\
& \sum_{j=1}^{m} \lambda_{j}=1, \quad \lambda_{1} \geqslant 0, \ldots, \lambda_{m} \geqslant 0 .
\end{aligned}
$$

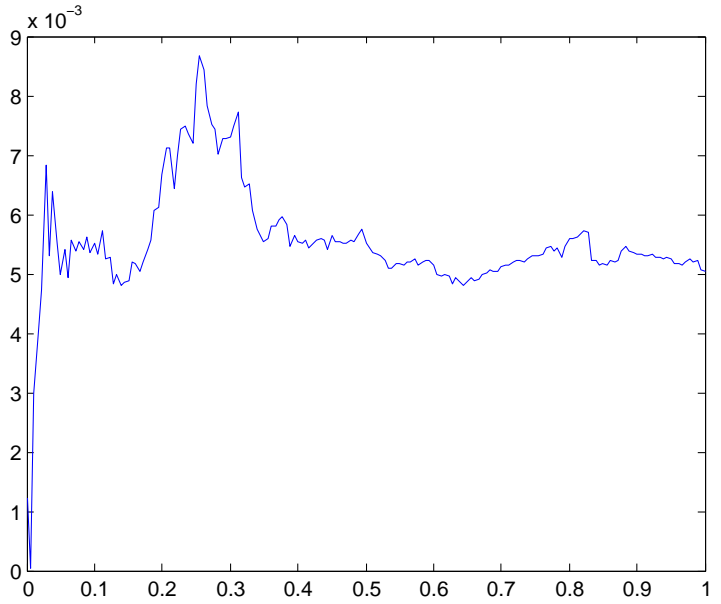

(a) weights in mixed CVaR (14)

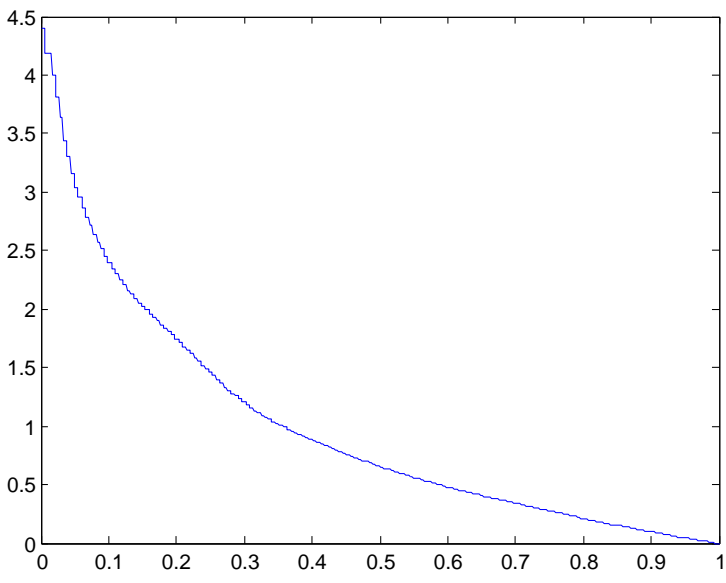

(b) weight function in the dual utility (6)

Figure 3: Law-invariant solutions of the inverse portfolio problem (19).

Example 4 Let $\Omega$ be finite uniform as in Example 1. Then Proposition 3 implies that a law-invariant solution $\rho$ to (19), if exists, can be found in the form (14) with $m=T$ and $\alpha_{j}=j / T, j=1, \ldots, T$, and with $\lambda_{1}, \ldots, \lambda_{T}$ found from (40). This $\rho$ can be equivalently represented in the spectral form (6) with $\phi(\alpha)=T \sum_{j=i}^{T} \lambda_{j} / j$ for $\alpha \in\left[\frac{j-1}{T}, \frac{j}{T}\right), j=1, \ldots, T$. If, in addition, the rate of return $X=\left(x_{1}, \ldots, x_{T}\right)$ of some instrument is required to be in the acceptance set of $\rho$, the system (40) includes extra constraint $\rho(X) \leqslant 0$. As in Example 1, let $n=92$ and $T=182$. 


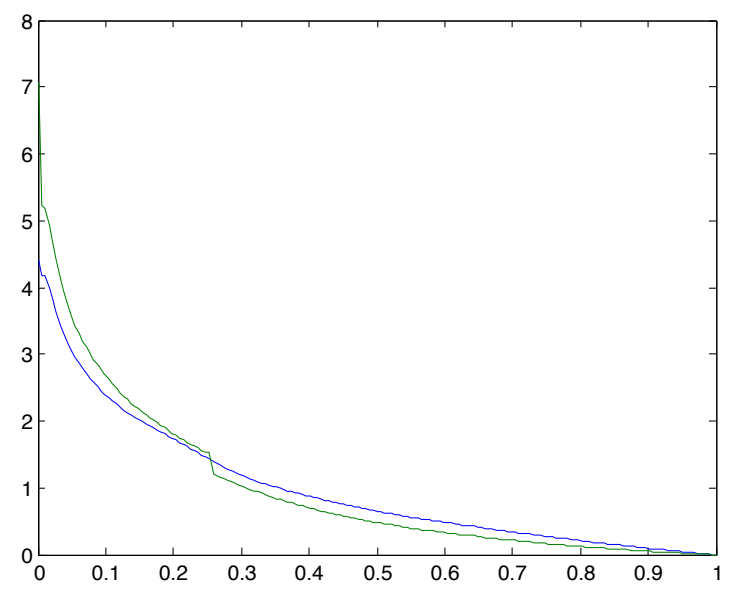

Figure 4: Change in the weight function in the dual utility (6) when $X=\frac{1}{n} \sum_{i=1}^{n} r_{i}$ is included in the acceptance set. The blue curve is the same as in Figure 3b, whereas the green one corresponds to the modified dual utility.

(i) For $X^{*}=\left(x_{1}^{*}, \ldots, x_{T}^{*}\right)$, being the rate of return of the optimal mean-variance portfolio (see Example 1(i)), the system (40) has a solution, and the found weights in mixed CVaR (14) are shown in Figure $3 a$, whereas the the weight function $\phi(\alpha)$ of the same solution in the dual utility form (6) is depicted in Figure $3 b$. Figure 4 shows how the weight function in the dual utility (6) changes when $X=\frac{1}{n} \sum_{i=1}^{n} r_{i}$ is included in the acceptance set (in this case, the system (40) includes extra contsraint $\rho(X) \leqslant 0$ ).

(ii) For $X^{*}=\left(x_{1}^{*}, \ldots, x_{T}^{*}\right)$, being the rate of return of the FTSE 100 index (see Example 1(ii)), the system (40) has no solution.

In general, the system (40) may have no solution in the following cases:

(a) If there exists $Y \in \mathcal{X}$ such that (24) holds, the inverse problem (19) has no solution in the form of mixed $\mathrm{CVaR}$ (5), and therefore, there is no solution in the form (14). This is the case in Example 4 (ii).

(b) For a general probability space, the inverse problem (19) may have no solution in the form (14) even if conditions (a)-(e) in Proposition 2 hold.

(c) If the probability space $\Omega$ is finite uniform with $T$ atoms, and the inverse problem (19) has a law-invariant solution, then the solution can be chosen in the form (14) with $m=T$ and $\alpha_{j}=j / T, j=1, \ldots, T$. Obviously, this does not apply when $m<T$.

In these cases, the investor may seek to find an approximate solution to the inverse problem (19). There are at least three different approximation approaches.

\subsection{Approximation in the space of risk measures}

Suppose conditions (a)-(d) in Proposition 2 hold, so that the inverse problem (19) has an exact solution $\rho^{*}$ in the form of mixed CVaR (5). Then an approximate solution $\rho$ in the form (14) with fixed $m$ can be found by minimizing the distance to $\rho^{*}$ in the space of risk measures:

$$
\min _{\rho \in \mathcal{R}} d\left(\rho^{*}, \rho\right),
$$

where $d(\cdot, \cdot)$ is a metric defined by

$$
d\left(\rho_{1}, \rho_{2}\right)=\sup _{X \in \mathcal{L}^{\infty}(\Omega):|X| \leqslant 1}\left|\rho_{1}(X)-\rho_{2}(X)\right|,
$$


and $\mathcal{R}$ is either the $2 m$-parameter family of all risk measures (14) with fixed $m$ or the $m$-parameter family of all risk measures (14) with fixed $m$ and fixed $\alpha_{1}, \ldots, \alpha_{m}$. In [12], the problem (41) was formualated for deviation measures $[27,28]$ and was reduced to finding a piecewise linear approximation of a convex function on $(0,1)$. The drawback of this approximation approach is that it requires knowing the exact solution $\rho^{*}$, which, moreover, may not exist.

\subsection{Approximation in the space of random variables}

An alternative formulation of the inverse problem (19) is: given optimal (preferred) rate of return $\widehat{X} \in \mathcal{X}$, find such $\rho \in \mathcal{R}$ that a solution $X^{*}$ of (16) has the least underperformance with respect to $\widehat{X}$ :

$$
\min _{\rho \in \mathcal{R}, X^{*} \in \mathcal{X}}\left\|\left[X^{*}-\widehat{X}\right]_{-}\right\|_{p} \quad \text { subject to } \quad X^{*} \in \underset{X \in \mathcal{X}}{\operatorname{argmin}} \rho(X),
$$

where $\mathcal{R}$ is the $m$-parameter family of all risk measures (14) with fixed $m$ and fixed $\alpha_{1}, \ldots, \alpha_{m},[t]_{-}=$ $\max \{0,-t\}$, and $\|\cdot\|_{p}$ is the $\mathcal{L}^{p}$-norm with some $p \in[1, \infty]$ (typically, $p=1$ or $p=\infty$ ). The objective function $\left\|\left[X^{*}-\widehat{X}\right]_{-}\right\|_{p}$ can be replaced by any other suitable function $f\left(\widehat{X}, X^{*}\right)$.

In fact, the problem (42) can be viewed as optimal tracking control, in which $\widehat{X}$ and $\rho$ are a target and a control "variable," respectively, or even as shape optimization, where $\lambda_{1}, \ldots, \lambda_{m}$ constitute the "risk shape." However, the disadvantage of the optimization problem (42) is that its objective value as a function of $\lambda_{1}, \ldots \lambda_{n}$ :

$$
f\left(\lambda_{1}, \ldots \lambda_{n}\right)=\min _{X^{*} \in \underset{X \in \mathcal{X}}{\operatorname{argmin}} \rho(X)}\left\|\left[X^{*}-\widehat{X}\right]_{-}\right\|_{p}
$$

is non-convex and even discontinuous. The following example shows that the function (43) has its minimum exactly at a point of removable discontinuity, which is unlikely to be found by standard optimization methods.

Example 5 Let $\Omega=\left(\omega_{1}, \omega_{2}\right)$ with $\mathbb{P}\left[\omega_{i}\right]=1 / 2, p=\infty, n=2, r_{0}=0, r_{1}=(0,0), r_{2}=(-1,2)$, $\widehat{X}=\frac{2}{3} r_{1}+\frac{1}{3} r_{2}=(-1 / 3,2 / 3), m=2, \alpha_{1}=0, \alpha_{2}=1, \lambda_{1}=\lambda, \lambda_{2}=1-\lambda$. Suppose short sales are not allowed. Then the objective function (43) is given by

$$
f(\lambda)=\left\{\begin{array}{l}
2 / 3, \quad \lambda \in[0,1 / 3) \cup(1 / 3,1] \\
0, \quad \lambda=1 / 3 .
\end{array}\right.
$$

Detail. In this case, mixed CVaR (14) takes the form $\rho(X)=\lambda_{1} \operatorname{CVaR}_{0}(X)+\lambda_{2} \operatorname{CVaR}_{1}(X)=\lambda(-\inf X)+$ $(1-\lambda)(-E[X]), \lambda \in[0,1]$, and $\mathcal{X}=\left\{X \mid(1-x) r_{1}+x r_{2}=(-x, 2 x), x \in[0,1]\right\}$, so that $\rho(X)=$ $\lambda(-(-x))+(1-\lambda)(-(x / 2))=x(3 \lambda-1) / 2$. Thus, $X^{*}=\left(-x^{*}, 2 x^{*}\right)$ is an optimal rate of return, where $x^{*}=1$ when $\lambda \in[0,1 / 3), x^{*} \in[0,1]$ when $\lambda=1 / 3$, and $x^{*}=0$ when $\lambda \in(1 / 3,1]$, and the optimal value of (43) is determined by $f(\lambda)=\left\|[(-1,2)-(-1 / 3,2 / 3)]_{-}\right\|_{\infty}$ when $\lambda \in[0,1 / 3), f(\lambda)=0$ when $\lambda=1 / 3$, and $f(\lambda)=\left\|[(0,0)-(-1 / 3,2 / 3)]_{-}\right\|_{\infty}$ when $\lambda \in(1 / 3,1]$, which simplifies to (44).

In the next section, the problem (42) is "modified" into a convex one: instead of minimizing distance in the space of r.v.'s, we can approximate the value of an optimal risk measure for a given $\widehat{X}$.

\subsection{Approximation of the optimal value}

For given $\widehat{X} \in \mathcal{X}$, a solution $\rho \in \mathcal{R}$ can be sought to minimize $\rho(\widehat{X})-\rho\left(X^{*}\right)$ for $X^{*} \in \operatorname{argmin}_{X \in \mathcal{X}} \rho(X)$ :

$$
\min _{\rho \in \mathcal{R}, X^{*} \in \mathcal{X}}\left(\rho(\widehat{X})-\rho\left(X^{*}\right)\right) \quad \text { subject to } \quad X^{*} \in \underset{X \in \mathcal{X}}{\operatorname{argmin}} \rho(X) .
$$

Let $\mathcal{X}$ be given by (17). Then with the optimality conditions (22), the problem (45) can be written as 


$$
\min _{\rho \in \mathcal{R}, X^{*} \in \mathcal{X}}\left(\rho(\widehat{X})-\rho\left(X^{*}\right)\right) \quad \text { subject to } \quad \exists Q \in \mathcal{Q}_{\rho}: E\left[r_{i} Q\right] \leqslant-\rho\left(X^{*}\right), \quad i=1, \ldots, n,
$$

where $\mathcal{Q}_{\rho}$ is the risk envelope of $\rho$. This problem is equivalent to

$$
\min _{\rho \in \mathcal{R}, X^{*} \in \mathcal{X}, Q \in \mathcal{Q}_{\rho}}\left(\rho(\widehat{X})-\rho\left(X^{*}\right)\right) \quad \text { subject to } \quad E\left[r_{i} Q\right] \leqslant-\rho\left(X^{*}\right), \quad i=1, \ldots, n,
$$

or, with a new variable $z=\rho(\widehat{X})-\rho\left(X^{*}\right)$, to

$$
\min _{z \in \mathbb{R}, \rho \in \mathcal{R}, X^{*} \in \mathcal{X}, Q \in \mathcal{Q}_{\rho}} z \quad \text { subject to } \quad z=\rho(\widehat{X})-\rho\left(X^{*}\right), \quad E\left[r_{i} Q\right]+\rho(\widehat{X})-z \leqslant 0, \quad i=1, \ldots, n .
$$

Suppose $\mathcal{R}$ is the family of all risk measures (14) with fixed $m$ and fixed $\alpha_{1}, \ldots, \alpha_{m}$. With $\Lambda=\{\lambda=$ $\left.\left(\lambda_{1}, \ldots, \lambda_{m}\right) \mid \sum_{i=1}^{m} \lambda_{i}=1, \lambda_{i} \geqslant 0, i=1, \ldots, m\right\}$, the problem (48) reduces to

$$
\min _{z \in \mathbb{R}, \lambda \in \Lambda, X^{*} \in \mathcal{X}, Q \in \mathcal{Q}_{\lambda}} z \quad \text { subject to } \quad z=\rho_{\lambda}(\widehat{X})-\rho_{\lambda}\left(X^{*}\right), \quad E\left[r_{i} Q\right]+\rho_{\lambda}(\widehat{X})-z \leqslant 0, \quad i=1, \ldots, n,
$$

where $\rho_{\lambda}(X)=\sum_{j=1}^{m} \lambda_{j}\left(\operatorname{CVaR}_{\alpha_{j}}(X)\right)$ and $\mathcal{Q}_{\lambda}$ is the corresponding risk envelope.

The variable $X^{*}$ in (49) is included only in the constraint $z=\rho_{\lambda}(\widehat{X})-\rho_{\lambda}\left(X^{*}\right)$ and can always be chosen to satisfy it, so that the problem (49) simplifies to

$$
\min _{z \in \mathbb{R}, \lambda \in \Lambda, Q \in \mathcal{Q}_{\lambda}} z \quad \text { subject to } E\left[r_{i} Q\right]+\rho_{\lambda}(\widehat{X})-z \leqslant 0, \quad i=1, \ldots, n,
$$

see Appendix A for the proof of the equivalence of (49) and (50).

The problem (50) has an interesting interpretation: it seeks to find a risk measure $\rho_{\lambda}$ for which the optimality conditions (22) can be satisfied for $\widehat{X}$ as accurately as possible.

Using (40), we can rewrite (50) as

$$
\begin{array}{ll}
\underset{\substack{z, \lambda_{1}, \ldots, \lambda_{m}, S_{1}, \ldots, S_{m}}}{\min } z \text { subject to } & \sum_{j=1}^{m} E\left[r_{i} S_{j}\right]+\sum_{j=1}^{m} \lambda_{j} \operatorname{CVaR}_{\alpha_{j}}(\widehat{X})-z \leqslant 0, \quad i=1, \ldots, n, \\
& E\left[S_{j}\right]=\lambda_{j}, \quad S_{j} \in\left[0, \lambda_{j} / \alpha_{j}\right], \quad j=1, \ldots, m, \\
& \sum_{j=1}^{m} \lambda_{j}=1, \quad \lambda_{1} \geqslant 0, \ldots, \lambda_{m} \geqslant 0 .
\end{array}
$$

For a discrete probability space, (51) simplifies to a linear program.

Example 6 In the settings of Example 1(ii), let $\mathcal{X}$ be a feasible set with no short positions:

$$
\mathcal{X}=\left\{X=\left(x_{1}, \ldots, x_{T}\right) \mid x_{t}=\sum_{i=1}^{n} r_{i t} v_{i}, \quad t=1, \ldots, T, \quad \sum_{i=1}^{n} v_{i}=1, v_{1} \geqslant 0, \ldots, v_{n} \geqslant 0\right\},
$$

$\widehat{X} \in \mathcal{X}$ be the rate of return of the FTSE 100 index, and $m=5, \alpha_{1}=0.01, \alpha_{2}=0.05, \alpha_{3}=0.2, \alpha_{4}=0.5$, and $\alpha_{5}=1$. Then optimal $\lambda_{1}, \ldots, \lambda_{5}$ in (14) can be found from (51) and yield

$$
\rho=0.011 \mathrm{CVaR}_{0.01}+0.008 \mathrm{CVaR}_{0.05}+0.069 \mathrm{CVaR}_{0.2}+0.149 \mathrm{CVaR}_{0.05}+0.763 \mathrm{CVaR}_{1} .
$$

Example 7 If the probability space $\Omega$ is finite uniform with $T$ atoms, $m=T$, and $\alpha_{j}=j / T, j=1, \ldots, T$, then the optimal value in (51) is zero if and only if condition (b) in Proposition 2 holds. In other words, (51) is a linear programming test to determine whether $\widehat{X}$ is SSD-efficient within $\mathcal{X}$. In contrast to the existing SSD-efficiency tests [10,17,24], (51) has the advantage to return an explicit optimal risk measure (or dual utility) for SSD-efficient portfolios as well as an "approximately optimal" risk measure for inefficient ones. In the later case, the optimal value in (51) can also be interpreted as a measure of SSD-inefficiency for $\widehat{X}$. 


\subsection{Geometric interpretation}

Let $\mathcal{Y} \subset \mathbb{R}^{m}$ be given by

$$
\mathcal{Y}=\left\{y=\left(y_{1}, \ldots, y_{m}\right) \mid \exists X \in \mathcal{X}: \mathrm{CVaR}_{\alpha_{j}}(X) \leqslant y_{j}, j=1, \ldots, m\right\} .
$$

Then the inverse portfolio problem (19) has an exact solution in the form (14) if and only if the point $y\left(X^{*}\right)=\left(\mathrm{CVaR}_{\alpha_{1}}\left(X^{*}\right), \ldots, \mathrm{CVaR}_{\alpha_{m}}\left(X^{*}\right)\right)$ lies on the boundary of $\mathcal{Y}$. Indeed, if $y\left(X^{*}\right)$ is in the interior of $\mathcal{Y}$, then $\mathrm{CVaR}_{\alpha_{j}}\left(X^{*}\right) \geqslant \mathrm{CVaR}_{\alpha_{j}}\left(X^{\prime}\right)+\epsilon, j=1, \ldots, m$, for some $X^{\prime} \in \mathcal{X}$ and $\epsilon>0$, so that $\rho\left(X^{*}\right) \geqslant \rho\left(X^{\prime}\right)+\epsilon$ for any $\rho$ admitting the form (14), hence, $X^{*}$ cannot be optimal. Conversely, if $y\left(X^{*}\right)$ is on the boundary of a (convex closed) set $\mathcal{Y}$, then there exists a supporting hyperplane for $\mathcal{Y}$ through $y\left(X^{*}\right)$, and the coefficients $\lambda_{1}, \ldots, \lambda_{m}$ in the equation of that hyperplane are exactly the coefficients of the desired risk measure $\rho$ in (14).

Now let $\widehat{X}$ be a portfolio such that $y(\widehat{X})$ is in the interior of $\mathcal{Y}$, so that an exact solution of the inverse problem (19) does not exist and we seek to find an approximate one. The problem (42) is then asks for a point on the boundary of $\mathcal{Y}$, which is "closest" to the given point $y(\widehat{X})$ in its interior, where a measure of closeness can be defined in different ways. In contrast, the problem (45) requires finding a hyperplane $\mathcal{A}$ that contains $y(\widehat{X})$ such that the distance from $\mathcal{A}$ to the supporting hyperplane parallel to $\mathcal{A}$ is as small as possible.

The first approach identifies a risk measure $\rho_{1}$ such that optimal portfolio with $\rho_{1}$ is as "close" to $\widehat{X}$ as possible, i.e. a similar portfolio is optimal, whereas the second approach finds a risk measure $\rho_{2}$, such that risk $\rho_{2}(\widehat{X})$ is as close to the optimal risk as possible, i.e. a given portfolio is almost optimal.

\section{Conclusions and Future Research}

This work has proposed to identify agent's risk preferences in the form of a coherent risk measure by solving the inverse portfolio problem in single-period and multi-period formulations. The negative coherent risk measure is viewed as agent's utility functional and can be recovered either through identifying a convex set of admissible probability measures (so-called risk envelope) or through finding either the risk profile in mixed CVaR or the weight function in negative Yaari's dual utility (the last two representations are law-invariant coherent risk measures and may not exist). The inverse portfolio problem assumes that the rate of return of the portfolio that the agent is most satisfied with minimizes the sought coherent risk measure and is given as a sequence of known random variables (r.v.'s) for the multi-period case (single r.v. for the single-period case). In Examples 1-4 and 6 , the coherent risk measure is recovered in all three forms based on the weekly historical rates of return of a portfolio formed by 92 stocks from the FTSE 100 index for the period from 3-January-2011 to 30-June-2014. As a future research, the suggested inverse portfolio problem approach can be extended to identifying risk preferences in the frameworks of other decision theories.

\section{Acknowledgment}

The authors are grateful to the anonymous referees for the comments and suggestions, which helped to improve the quality of the paper. The first author also thanks the University of Leicester for granting him the academic study leave to do this research.

\section{A Appendix: Proof of the equivalence of (49) and (50)}

Let $\left(z_{1}, \lambda_{1}, X_{1}^{*}, Q_{1}\right)$ and $\left(z_{2}, \lambda_{2}, Q_{2}\right)$ be optimal solutions in (49) and (50), respectively, with $\rho_{1}$ and $\rho_{2}$ being the corresponding risk measures.

First, we prove that

$$
z_{1} \leqslant \rho_{\lambda}(\widehat{X})-\rho_{\lambda}\left(X_{1}^{*}\right) \quad \forall \lambda \in \Lambda
$$


Indeed, the original problem (45) can be rewritten as

$$
\min _{\lambda \in \Lambda} \max _{X \in \mathcal{X}}\left(\rho_{\lambda}(\widehat{X})-\rho_{\lambda}(X)\right)
$$

For a fixed $X, \rho_{\lambda}(\widehat{X})-\rho_{\lambda}(X)$ is a linear function with respect to $\lambda$, and for a fixed $\lambda$, it is a concave function with respect to $X$. Hence, by Sion's minimax theorem [33], the optimal value of (53) is equal to that of the problem

$$
\max _{X \in \mathcal{X}} \min _{\lambda \in \Lambda}\left(\rho_{\lambda}(\widehat{X})-\rho_{\lambda}(X)\right)=\max _{X \in \mathcal{X}} \min _{\lambda \in \Lambda} \sum_{j=1}^{m} \lambda_{j}\left(\operatorname{CVaR}_{\alpha_{j}}(\widehat{X})-\operatorname{CVaR}_{\alpha_{j}}(X)\right)
$$

Observe that $\min _{\lambda \in \Lambda} \sum_{j=1}^{m} \lambda_{j}\left(\mathrm{CVaR}_{\alpha_{j}}(\widehat{X})-\mathrm{CVaR}_{\alpha_{j}}(X)\right)$ is an unweighted version of a continuous knapsack problem, and [3, Proposition 17.1] implies that (54) simplifies to

$$
\max _{X \in \mathcal{X}} \min _{j \in\{1, \ldots, m\}}\left(\operatorname{CVaR}_{\alpha_{j}}(\widehat{X})-\operatorname{CVaR}_{\alpha_{j}}(X)\right),
$$

or, equivalently, to

$$
\max _{X \in \mathcal{X}, z \in \mathbb{R}}-z \quad \text { subject to } \quad-z \leqslant \operatorname{CVaR}_{\alpha_{j}}(\widehat{X})-\operatorname{CVaR}_{\alpha_{j}}(X), \quad j=1, \ldots, m .
$$

Since $z_{1}$ is the optimal value of (49), the problem (55) implies that

$$
z_{1} \leqslant \mathrm{CVaR}_{\alpha_{j}}(\widehat{X})-\mathrm{CVaR}_{\alpha_{j}}\left(X_{1}^{*}\right), \quad j=1, \ldots, m,
$$

and (52) follows.

Now we prove the equivalence of (49) and (50). Since $\left(z_{1}, \lambda_{1}, Q_{1}\right)$ is a feasible solution to (50), $z_{2} \leqslant z_{1}$. By contradiction, let $z_{2}<z_{1}$. In addition,

$$
z_{2} \geqslant E\left[\widehat{X} Q_{2}\right]+\rho_{2}(\widehat{X}) \geqslant 0,
$$

where the first and second inequalities follow from the constraints in (50) and from $Q_{2} \in \mathcal{Q}_{\lambda_{2}}$, respectively. Then $f(\beta)=\rho_{2}(\widehat{X})-\rho_{2}\left(\beta \widehat{X}+(1-\beta) X_{1}^{*}\right), \beta \in[0,1]$, is a continuous function with $f(0) \geqslant z_{1}$ by (52) and with $f(1)=0$, so that $f\left(\beta^{*}\right)=z_{2}$ for some $\beta^{*} \in[0,1]$. Then $z_{2}=\rho_{2}(\widehat{X})-\rho_{2}\left(X_{2}^{*}\right)$ for $X_{2}^{*}=$ $\beta^{*} \widehat{X}+\left(1-\beta^{*}\right) X_{1}^{*} \in \mathcal{X}$. Thus, $\left(z_{2}, \lambda_{2}, X_{2}^{*}, Q_{2}\right)$ is a feasible solution in (49) that yields $z_{1} \leqslant z_{2}$, which contradicts the assumption that $z_{2}<z_{1}$.

\section{References}

[1] M. Allais. Le comportement de l'homme rationnel devant le risque: Critique des postulats et axiomes de l'ecole americaine. Econometrica, 21(4):503-546, 1953.

[2] P. Artzner, F. Delbaen, J.-M. Eber, and D. Heath. Coherent measures of risk. Mathematical Finance, 9:203-227, 1999.

[3] J.Vygen B. Konte. Combinatorial Optimization: Theory and Algorithms, volume 21 of Algorithms and Combinatorics. Springer, 2012.

[4] N. Bäuerle and A. Müller. Stochastic orders and risk measures: Consistency and bounds. Insurance: Mathematics and Economics, 38:132-148, 2006. 
[5] D. E. Bell. Disappointment in decision making under uncertainty. Operations Research, 33(1):1-27, 1985.

[6] R.-A. Dana. A representation result for concave Schur-concave functions. Mathematical Finance, 15(4):613-634, 2005.

[7] P. C. Fishburn. Transitive measurable utility. Journal of Economic Theory, 31:293-317, 1983.

[8] H. Föllmer and A. Schied. Stochastic Finance. de Gruyter, Berlin New York, 3 edition, 2011.

[9] C. R. Fox and R. A. Poldrack. Prospect theory and the brain. In P. W. Glimcher, C. F. Camerer, E. Fehr, and R. A. Poldrack, editors, Neuroeconomics, pages 145-173. Academic Press, London, 2009.

[10] B. Grechuk. A simple ssd-efficiency test. Optimization Letters, 8(7):2135-2143, 2014.

[11] B. Grechuk, A. Molyboha, and M. Zabarankin. Mean-deviation analysis in the theory of choice. Risk Analysis: An International Journal, 32(8):1277-1292, 2012.

[12] B. Grechuk and M. Zabarankin. Inverse portfolio problem with mean-deviation model. European Journal of Operational Research, 234(2):481-490, 2014.

[13] S. Guriev. On microfoundations of the dual theory of choice. The Geneva papers on risk and insurance theory, 26(2):117-137, 2001.

[14] E. Jouini, W. Schachermayer, and N. Touzi. Optimal risk sharing for law invariant monetary utility functions. Mathematical Finance, 18(2):269-292, 2008.

[15] D. Kahneman and A. Tversky. Prospect theory: An analysis of decision under risk. Econometrica, 47:263-291, 1979.

[16] D. Kahneman and A. Tversky. Advances in prospect theory: Cumulative representation of uncertainty. Journal of Risk and Uncertainty, 5:297-323, 1992.

[17] M. Kopa and P. Chovanec. A second-order stochastic dominance portfolio efficiency measure. Kybernetika, 44(2):243-258, 2008.

[18] S. Kusuoka. On law invariant coherent risk measures. Advances in Mathematical Economics, 3:83-95, 2001.

[19] J. Leitner. A short note on second-order stochastic dominance preserving coherent risk measures. Mathematical Finance, 15(4):649-651, 2005.

[20] V. Lesnevski, B. L. Nelson, and J. C. Staum. Simulation of coherent risk measures based on generalized scenarios. Management Science, 53(11):1756-1769, 2007.

[21] G. Loomes and R. Sugden. Regret theory: An alternative theory of rational choice under uncertainty. Economic Journal, 92(4):805-824, 1982.

[22] S. O. Lozza, H. Shalit, and F. J. Fabozzi. Portfolio selection problems consistent with given preference orderings. International Journal of Theoretical and Applied Finance, 16(5), 2013.

[23] N. Miller and A. Ruszczynski. Risk-adjusted probability measures in portfolio optimization with coherent measures of risk. European Journal of Operational Research, 191(1):193-206, 2008.

[24] T. Post. Empirical tests for stochastic dominance efficiency. Journal of Finance, 58:1905-1932, 2003. 
[25] J. Quiggin. Generalized Expected Utility Theory. The Rank-Dependent Model. Kluwer Academic Publishers, Boston, 1993.

[26] R. T. Rockafellar and S. Uryasev. Conditional value-at-risk for general loss distributions. Journal of Banking and Finance, 26(7):1443-1471, 2002.

[27] R. T. Rockafellar, S. Uryasev, and M. Zabarankin. Deviation measures in risk analysis and optimization. Technical Report 2002-7, Industrial and Systems Engineering Department, University of Florida, 2002.

[28] R. T. Rockafellar, S. Uryasev, and M. Zabarankin. Generalized deviations in risk analysis. Finance and Stochastics, 10(1):51-74, 2006.

[29] R. T. Rockafellar, S. Uryasev, and M. Zabarankin. Master funds in portfolio analysis with general deviation measures. The Journal of Banking and Finance, 30(2):743-778, 2006.

[30] R. T. Rockafellar, S. Uryasev, and M. Zabarankin. Optimality conditions in portfolio analysis with general deviation measures. Mathematical Programming, 108(2-3):515-540, 2006.

[31] A. Röell. Risk aversion in quiggin and yaari's rank-order model of choice under uncertainty. The Economic Journal, 97(Supplement: Conference Papers):143-159, 1987.

[32] B. Rudloff. Coherent hedging in incomplete markets. Quantitative Finance, 9(2):197-206, 2009.

[33] M. Sion. On general minimax theorems. Mathematical Finance, 8(1):171-176, 1958.

[34] J. von Neumann and O. Morgenstern. Theory of Games and Economic Behavior. Princeton University Press, Princeton, NJ, 3 edition, 1953.

[35] M. E. Yaari. The dual theory of choice under risk. Econometrica, 55:95-115, 1987.

[36] M. Zabarankin and S. Uryasev. Statistical Decision Problems: Selected Concepts and Portfolio Safeguard Case Studies. Springer, Berlin, 2014.

[37] C. Zalinescu. Convex Analysis in General Vector Spaces. World Scientific Publishing, Singapore, 2002. 